\title{
Renormalization of the Higgs Model: Minimizers, Propagators and the Stability of Mean Field Theory *
}

Tadeusz Bałaban ${ }^{\star}$, John Imbrie, and Arthur Jaffe

Lyman Laboratory of Physics, Harvard University, Cambridge, MA 02138, USA

Dedicated to the memory of Kurt Symanzik

\begin{abstract}
We study the effective actions $S^{(k)}$ obtained by $k$ iterations of a renormalization transformation of the U(1) Higgs model in $d=2$ or 3 spacetime dimensions. We identify a quadratic approximation $S_{Q}^{(k)}$ to $S^{(k)}$ which we call mean field theory, and which will serve as the starting point for a convergent expansion of the Green's functions, uniformly in the lattice spacing. Here we show how the approximations $S_{0}^{(k)}$ arise and how to handle gauge fixing, necessary for the analysis of the continuum limit. We also establish stability bounds on $S_{Q}^{(k)}$, uniformly in $k$. This is an essential step toward proving the existence of a gap in the mass spectrum and exponential decay of gauge invariant correlations.
\end{abstract}

\section{Table of Contents}

1. The Model . . . . . . . . . . . . . . . . . . . . . . . . . . 300

2. Block Fields and Scaling . . . . . . . . . . . . . . . . . . . 302

3. The First Renormalization Step . . . . . . . . . . . . . . . . . 306

4. Form of the $k^{\text {th }}$ Effective Action . . . . . . . . . . . . . . . 308

4.1. The Axial Gauge Propagator and Minimizer . . . . . . . . . . 309

4.2. The Quadratic Action and Plaquette Fields . . . . . . . . . . 310

4.3. Quadratic Action for Curls . . . . . . . . . . . . . . . . . 311

4.4. Landau Gauge Propagator and Minimizer . . . . . . . . . . . 311

4.5. Bond Fields . . . . . . . . . . . . . . . . . . . . . . . . 312

4.6. The Scalar Field Action . . . . . . . . . . . . . . . . . . . . . . 313

5. Relations Among Minimizers and Propagators . . . . . . . . . . . . 313

5.1. Change of Gauge for Minimizers . . . . . . . . . . . . . . . . . 313

5.2. Decomposition of Axial Gauge Green's Functions. . . . . . . 315

5.3. Minimizers and Green's Functions . . . . . . . . . . . . . . 316

* Supported in part by the National Science Foundation under Grant PHY 82-03669

* Current address: Department of Mathematics, University of Michigan, Ann Arbor, MI 48109, USA 
6. Renormalization Transformations Produce the Effective Action as Claimed . . . . . . . . . . . . . . . . . . . . . . . . . . 317

6.1. $\sigma_{k}=\sigma_{k+1}+$ Fluctuation Form . . . . . . . . . . . . . . . 318

6.2. $u_{k}=u_{k+1}$. Fluctuation (Modulo Gauge Transformation) . . . . . 319

6.3. Scalar Field Renormalization. . . . . . . . . . . . . . . . . 320

7. Positivity and Localization of the Effective Action. . . . . . . . . . 321

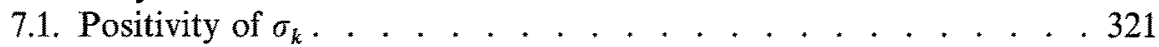

7.2. Regularity and Decay of Propagators and Minimizers . . . . . . 325

7.3. Positivity of $\Delta_{k}\left(u_{k}\right) \ldots \ldots \ldots$

Appendix: Quadratic Forms . . . . . . . . . . . . . . . 326

References . . . . . . . . . . . . . . . . . 329

\section{The Model}

We study the Higgs-type model of a U(1) gauge field $u$ coupled to a complex scalar field $\phi$ with a quartic self interaction. The total action functional is given on the unit lattice as

$$
S(u, \phi)=\sum_{p} e(\varepsilon)^{-2}(1-\operatorname{Re} u(p))+\frac{1}{2} \sum_{b}\left|\left(D_{u} \phi\right)_{b}\right|^{2}+\sum_{x} P\left(\phi_{x}\right)+E .
$$

The relation between the $\varepsilon$-lattice and unit-lattice actions is described in the Cargèse lectures [1], as well as a general introduction to mass generation in this model. This approximation with $\operatorname{Re} u$ is called the Wilson approximation to the continuum action.

The lattice points $x \in Z^{d}$ are endpoints of directed, unit length lattice bonds $b=\left(b_{-}, b_{+}\right)$which bound unit squares (plaquettes) denoted by $p$. The gauge field $u$ : bonds $\rightarrow \mathrm{U}(1)$, and

$$
u(p) \equiv \prod_{b \in \partial \partial p} u_{b} .
$$

Furthermore, we assume $b^{-1}=\left(b_{+}, b_{-}\right)$and $u_{b}^{-1}=u_{b^{-1}}$. The covariant derivative $D_{u} \phi$ is defined by $\left(D_{u} \phi\right)_{b}=u_{b} \phi_{b_{+}}-\phi_{b_{-}}$, and the coupling constant $e(\varepsilon)$ is $e(\varepsilon)=e \varepsilon^{(4-d) / 2}$. We consider $d=2$ or 3 space-time dimensions, in which case the self interaction is

$$
\mathscr{P}(\xi)=\lambda(\varepsilon)|\xi|^{4}-\frac{1}{4}\left(m^{2}+\delta m^{2}\right) \varepsilon^{2}|\xi|^{2},
$$

where $\lambda(\varepsilon)=\lambda \varepsilon^{4-d}$. The mass shift is consistent with perturbation theory with

$$
\delta m^{2}(\varepsilon)= \begin{cases}O\left(\ln \varepsilon^{-1}\right), & d=2 \\ O\left(\varepsilon^{-1}\right), & d=3\end{cases}
$$

The constant $E=E(e, \lambda, \varepsilon, A)$ is chosen to include vacuum energy normalization and renormalization counterterms from perturbation theory.

The continuum limit of the model with the action (1.1) has not yet been proved to exist, but our methods should lead to that result. The continuum limit of another action, the so-called Gaussian action, has been extensively studied. The Gaussian action is obtained by replacing $\operatorname{Re} u(p)$ in (1.1) with its quadratic 
approximation in Lie algebra variables. In two dimensions the existence theorem for the Gaussian action was established in [2]. In three dimensions and with a massive gauge field, the fundamental stability bounds were proved in [3] and the existence theorems based on these bounds are deduced in [4]. The infinite volume limit in these papers was analyzed using correlation inequalities, a method which is well suited for establishing existence theorems but which has not yet led to results on the nature of the spectrum in the resulting model. A lattice cutoff version of our spectral results is given in [5], where we also discuss the connection between Gaussian and Wilson actions on a lattice.

Our program is to study the Wilson action using a cluster expansion method. In this way we expect to both prove the existence of the theory and to establish properties of the particle spectrum. We expect that the Wilson action yields the same limiting continuum field theory as the Gaussian action. Hence the resulting invariance properties of the ground state and the properties of the particle spectrum which are consequences of the cluster expansion will also extend to the Gaussian model constructed previously. The Wilson action furthermore gives a natural setting for a possible generalization of these results to a nonabelian gauge model.

The basic strategy to gain control over the spectrum is to establish exponential decay of gauge invariant correlations. We develop a method of steepest descent to study integrals of the form

$$
\int \psi e^{-s} \mathscr{D} u \mathscr{D} \phi / \int e^{-s} \mathscr{D} u \mathscr{D} \phi,
$$

where $\psi$ is a gauge invariant functional of $u$ and $\phi$. Thus we need to define an expansion $S(u, \phi)=S\left(u_{c l}, \phi_{c l}\right)+\ldots$ in terms of a classical field $\left(u_{c l}, \phi_{c l}\right)$ and a fluctuation field $\left(u u_{c l}^{-1}, \phi-\phi_{c l}\right)$. We obtain this expansion in a sequence of steps, by studying block spin renormalization transformations of $S(u, \phi)$.

For most of this paper, we are concerned with identifying $S\left(u_{c l}, \phi_{c l}\right)$ and determining its properties. In particular, we find a quadratic action $S_{Q}$ which is close to $S\left(u_{c l}, \phi_{c l}\right)$. The transformation $S \rightarrow S_{Q}$ is an approximation to our renormalization transformation. We study the quadratic terms which arise by iteration of the renormalization transformation, yielding $S_{Q_{1}}, S_{Q_{2}}, \ldots, S_{Q_{k}}$, and establish uniform stability estimates (strictly positive lower bounds) on the $S_{Q}$ 's. These quadratic forms dominate the action in the "small field" region. In other papers we estimate the corrections due to large fields and show that they do not affect the basic picture. The end result is exponential decay of gauge invariant correlations, and a mass gap for both the Higgs particle and the photon.

Let us return to the action (1.1). The formal picture depends upon relating the action $S$ to quadratic actions $S_{Q}$. Note that we can define the plaquette field

$$
f_{p}=(i e(\varepsilon))^{-1} \ln u(p),
$$

so for small $f_{p}$,

$$
\sum_{p} e(\varepsilon)^{-2}(1-\operatorname{Re} u(p))=\sum_{p} \frac{1}{2} f_{p}^{2}+O\left(e(\varepsilon)^{2}\right) .
$$

The error term vanishes pointwise as $\varepsilon \rightarrow 0$ for $d<4$, and shows that the action (1.1) is the formally correctly scaled unit lattice action to study the continuum limit 
$\varepsilon \rightarrow 0$. In this paper we establish stability properties of various quadratic approximations to $S$. We use these later to establish properties of integrals (1.4).

\section{Block Fields and Sealing}

We study block spin renormalization of the Wilson action. This involves defining averages of $u$ and $\phi$ in lattice blocks, and performing the integral (1.4) conditioned by the block averages taking given values. In physics terminology we integrate high momentum degrees of freedom while fixing low momentum degrees of freedom in each block. If the blocks have side length $L$, a small positive integer, then we obtain functions on an $L Z^{d}$ lattice. Repeating this process leads us to study lattices $Z^{d}, L Z^{d}, L^{2} Z^{d}, \ldots, L^{k} Z^{d}, \ldots$. Since after each step it is often convenient to scale the $L Z^{d}$ lattice back to the unit lattice, we consider lattices with spacing $a$, with

$$
a=\varepsilon, \ldots, L^{-k}, \ldots, L^{-1}, 1, L .
$$

The natural $L^{2}$ inner product for spacing a comes from the norm (for scalar functions on the $a$-lattice),

$$
\|\phi\|_{a}^{2}=\sum_{i \in T_{a}}\left|\phi_{i}\right|^{2} a^{d}
$$

Here $T_{a}$ denotes a lattice with spacing $a$ and periodic (toroidal) boundary conditions. Generally we take $a=1, L$, or $L^{-k}$, the latter arising from $k$-fold scalings of an $L$-lattice to a unit lattice, following a block spin transformation. We also use lattices

$$
T_{a}^{(k)}=T_{a L^{-k}} \cap a Z^{d}
$$

Averaging operators map functions on the $T_{a}$ lattice to functions on the $T_{L a}$ lattice. We specify the map from the unit-lattice to the $L$-lattice, but this definition extends naturally from an $a$-lattice to an $\mathrm{La}$-lattice. We assume this extension in dealing with powers of averaging operators, etc.

Consider the example of a scalar field. Imbed the unit lattice in an $L$-lattice of block $B(y)$. Here $y=L n$ denotes a corner of a block, and $n \in Z^{d}$. Then $B(y)$ consists of points $x=\left(x_{1}, \ldots, x_{d}\right)$ such that

$$
L n_{j} \leqq x_{j}<L\left(n_{j}+1\right), \quad j=1,2, \ldots, d .
$$

Let $\Gamma$ denote a path (contour) composed of bonds, and define

$$
u(\Gamma)=\prod_{b \in \Gamma} u_{b}
$$

Contours $\Gamma_{1}, \Gamma_{2}$ can be composed if the endpoint of $\Gamma_{2}$ coincides with the starting point of $\Gamma_{1}$, so $u\left(\Gamma_{1} \circ \Gamma_{2}\right)=u\left(\Gamma_{1}\right) u\left(\Gamma_{2}\right)$.

We require a set of standard contours $\Gamma_{y x}$ from corners $y$ of blocks $B(y)$ to points $x \in B(y)$. Define $\Gamma_{y x}$ as the path obtained by following the coordinate axis one, then axis two, ..., etc., in going from $x$ to $y$. For example, if $x \in B(y)$, then

$$
x-y=\sum_{i=1}^{d} n_{i} e_{i}
$$


where $e_{i}$ denote unit vectors in the $i^{\text {th }}$ coordinate directions [and $n_{i} \geqq 0$ as $x \in B(y)$ ]. The path $\Gamma_{y x}$ is the path of length $n_{1}$ in the direction $-e_{1}$ followed by $n_{2}$ bonds in direction $-e_{2}$, etc. Let $\Gamma^{-1}$ denote the reverse of the path $\Gamma$. For a point $y^{\prime}$ which is a corner of an adjacent block, let $\Gamma_{y y^{\prime}}$ denote the $L$-lattice bond from $y$ to $y^{\prime}$.

The average $Q \phi$ of $\phi$ is defined by

$$
(Q \phi)_{y}=L^{-d} \sum_{x \in B(y)} u\left(\Gamma_{y x}\right) \phi_{x} .
$$

An important property of the average is that $Q$ commutes with gauge transformations. In other words, let $h$ denote a map from the unit lattice to $U(1)$. Then $h$ defines the gauge transformation

$$
\phi_{y} \rightarrow h(y) \phi_{y} \equiv \phi_{y}^{h}, \quad u_{b} \rightarrow h\left(b_{-}\right) h\left(b_{+}\right)^{-1} u_{b}=u_{b}^{h} .
$$

Clearly

$$
(Q \phi)^{h}=Q \phi^{h}
$$

A further property of $Q$ is that

$$
Q Q^{*}=I,
$$

where $Q^{*}$ is the adjoint in the scalar product (2.2). Thus $Q^{*}$ maps functions on the $a$-lattice into functions on the $L^{-1} a$ lattice. Furthermore $Q^{*} Q$ is an orthogonal projection. In other words $Q$ is a partial isometry.

One choice of gauge is especially good for algebraic operations, namely axial gauge. In axial gauge we choose $h$ in order to set $u_{b}=1$ for every $b$ which occurs in some $\Gamma_{y x}$. In other words, we specify a maximal tree $T(y)$ in each block $B(y)$ composed of bonds in any $\Gamma_{y x}, x \in B(y)$.

In the axial gauge, $Q \phi$ reduces to the ordinary average of $\phi$, and $Q^{*} Q$ is the projection onto $\phi$ which are constant on blocks $B(y)$.

Let us next consider the average of a gauge field $u$. If $b^{\prime}=y y^{\prime}$ is a bond on the $L$-lattice, then $\Gamma_{y y^{\prime}}=b^{\prime}$ and we average over contours $\Gamma_{x x^{\prime}}$ which are translates of $\Gamma_{y y^{\prime}}$, i.e. $x-x^{\prime}=y-y^{\prime}$. More specifically, define $Q$ on gauge fields on the unit lattice by

$$
(Q u)_{y y^{\prime}}=u\left(\Gamma_{y y^{\prime}}\right) \exp \left[L^{-d} \sum_{x \in B(y)} \ln u\left(\Gamma_{y x^{\prime}} \circ \Gamma_{x x^{\prime}} \circ \Gamma_{y^{\prime} x^{\prime}}^{-1} \circ \Gamma_{y^{\prime} y}\right)\right] .
$$

In (2.10) choose the logarithm so that

$$
-\pi \leqq \arg \ln u<\pi .
$$

With this choice, the discontinuity of $\ln u$ occurs at $u=-1$, namely the region of largest action. This is also the region of maximum suppression of probability, and hence the discontinuity will be seen not to affect integrals (1.4) in an important manner.

The average (2.10) is highly nonlinear. We also desire a linear average, defined on Lie algebra variables. Suppose that we can define

$$
A_{b}=(i e(\varepsilon))^{-1} \ln u_{b} \text {. }
$$




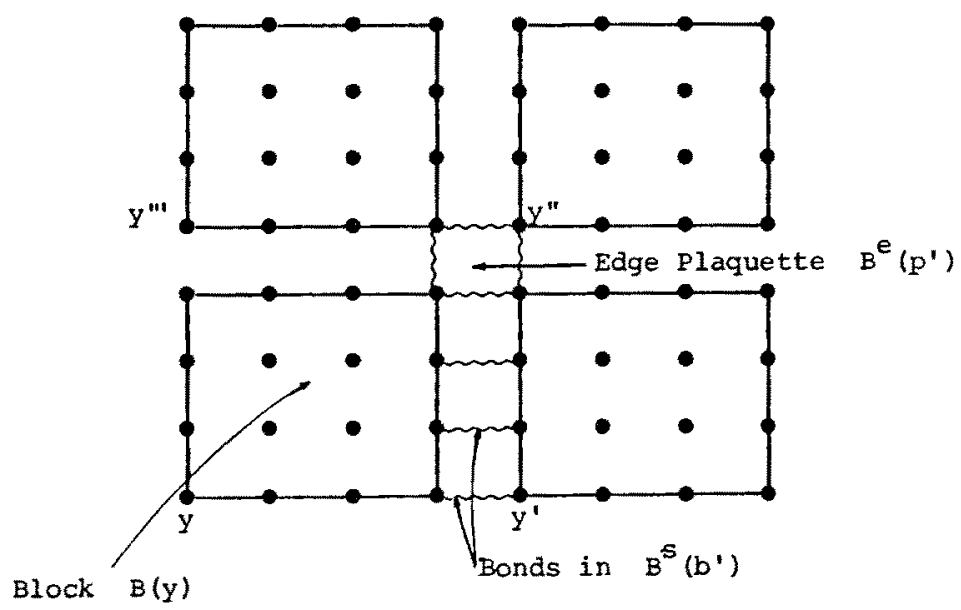

Fig. 1. Block variables for gauge fields, drawn in $d=2$. Four blocks are illustrated. Let $B(y)$ denote a block with $L=4$. The $L$-lattice bonds illustrated are $b^{\prime}=y y^{\prime}, y^{\prime} y^{\prime \prime}, y^{\prime \prime} y^{\prime \prime \prime}$, and $y^{\prime \prime \prime} y^{\prime}$. These four $L$-bonds bound a plaquette $p^{\prime}$. The corresponding edge plaquette $B^{\prime}\left(p^{\prime}\right)$ is indicated. The bond $b^{\prime}$ has associated surface bonds $B^{s}\left(b^{\prime}\right)$ which connect $B(y)$ and $B\left(y^{\prime}\right)$. A few bonds connecting distinct blocks are indicated by wavey lines

For $b^{\prime}=\left(b_{-}^{\prime}, b_{+}^{\prime}\right)$ a (directed) $L$-lattice bond from $b_{-}^{\prime}$ to $b_{+}^{\prime}$, we define the average

$$
(Q A)_{b^{\prime}}=L^{-(d+1)} \sum_{x \in B\left(b^{\prime}\right)} \sum_{b \in \Gamma_{x x^{\prime}}} A_{b},
$$

where $\Gamma_{x x^{\prime}}$ is the special contour from $x$ to $x^{\prime}$ and $x x^{\prime}$ is the parallel transport of the bond $b^{\prime}$ to start at $x$. Thus the bonds $b$ which enter the sum (2.13) range over the interior of the two $L$-blocks $B\left(b_{-}^{\prime}\right)$ and $B\left(b_{+}^{\prime}\right)$, as well as the (surface) bonds connecting these blocks.

The natural inner product on functions on bonds is

$$
\langle A, B\rangle_{a}=\sum_{b} a^{d} A_{b} B_{b} .
$$

We do not explicitly compute the adjoint $Q^{*}$. However, we now study related averages on surface bonds which connect adjacent blocks and on edge plaquettes which intersect four blocks.

Define the set of surface bonds $B^{s}\left(b^{\prime}\right)$ for an $L$-lattice bond $b^{\prime}$ as follows:

$$
B^{s}(b)=\left\{b: b=\left(b_{-}, b_{+}\right), b_{-} \in B\left(b_{-}^{\prime}\right), b_{+} \in B\left(b_{+}^{\prime}\right)\right\}
$$

(see Fig. 1). Associated with the surface is the average

$$
\left(Q^{s} A\right)_{b^{\prime}}=L^{-(\dot{d}-1)} \sum_{b \in B^{s}\left(b^{\prime}\right)} A_{b} \text {. }
$$

Then

$$
\left(Q^{s *} A\right)_{b}= \begin{cases}L A_{b^{\prime}}, & \text { if } b \in B^{s}\left(b^{\prime}\right), \\ 0, & \text { otherwise, }\end{cases}
$$

using the inner product (2.14). Also

$$
Q^{s} Q^{s *}=L I, \quad Q^{s *} Q^{s}=L P^{s},
$$


where $P^{s}$ is the projection onto configurations which are constant on surface bonds and zero on interior bonds. Furthermore, by (2.13),

$$
Q Q^{s *}=I \text {. }
$$

Finally, we define an edge average $Q^{e}$ associated with lattice plaquettes. The natural scalar product for functions on lattice plaquettes is

$$
\langle f, g\rangle_{a}=\sum_{p} a^{d} f_{p} g_{p}
$$

where $p$ is a plaquette on the $a$-lattice. Let $p^{\prime}$ denote an $L$-lattice plaquette. The set of edge plaquettes $B^{e}(p)$ are defined as those unit plaquettes $p$ which (i) are parallel to $p^{\prime}$, (ii) whose bonds touch four distinct $L$-blocks, and (iii) such that these $L$-blocks contain the corners of $p^{\prime}$ (see Fig. 1). The edge average $Q^{e}$ is defined by

$$
\left(Q^{e} f\right)\left(p^{\prime}\right)=L^{-(d-2)} \sum_{p \in B\left(p^{\prime}\right)} f(p) .
$$

Then

$$
\left(Q^{e *} f\right)(p)= \begin{cases}L^{2} f\left(p^{\prime}\right), & \text { if } p \in B^{e}\left(p^{\prime}\right), \\ 0, & \text { otherwise. }\end{cases}
$$

Also

$$
Q^{e} Q^{e *}=L^{2} I, \quad Q^{e *} Q^{e}=L^{2} P^{e},
$$

where $P^{e}$ is the orthogonal projection onto configurations which are constant on edge plaquettes and zero elsewhere.

We also need the $k$-fold averaging operators $Q_{k} \equiv(Q)^{k}$, etc. Especially important are $Q_{k}^{e} \equiv\left(Q^{e}\right)^{k}$ and $Q_{k}^{s}=\left(Q^{s}\right)^{k}$ which satisfy

where $\eta=L^{-k}$.

$$
Q_{k}^{e} Q_{k}^{e *}=L^{2 k} I=\eta^{-2} I, \quad Q_{k}^{s} Q_{k}^{s *}=L^{k} I=\eta^{-1} I,
$$

Finally we consider a scale transformation $\mathscr{S}_{L^{-1}}$ which maps $L$-lattice fields to unit lattice fields. The canonical scaling for scalar fields

$$
\left(\mathscr{S}_{L^{-1}} \phi\right)_{x}=L^{-(d-2) / 2} \phi_{L x}
$$

For gauge fields, the $L$-lattice action

$$
\sum_{p} L^{d}\left\langle f_{p}, \sigma f_{p}\right\rangle
$$

can be written in terms of a unit lattice action with

$$
\left(\mathscr{S}_{L^{-1}} f\right)_{p}=L^{d / 2} f_{L p}
$$

so for a quadratic form $\sigma$,

$$
\sum_{p \in T_{L}} L^{d}\left\langle f_{p}, \sigma f_{p}\right\rangle=\sum_{p \in T_{1}}\left\langle\left(\mathscr{S}_{L^{-1}} f\right)_{p}, \sigma\left(\mathscr{S}_{L^{-1}} f\right)_{p}\right\rangle .
$$

Then the $\varepsilon$-lattice action

$$
S_{\varepsilon}=\sum_{p \in T_{\varepsilon}} e^{-2} \varepsilon^{d-4}(1-\operatorname{Re} u(p))+\frac{1}{2} \sum_{b \in T_{s}} \varepsilon^{d}\left|D_{u} \phi\right|^{2}+\sum_{x \in T_{\varepsilon}} \varepsilon^{d} \mathscr{P}_{s}\left(\phi_{x}\right)+E,
$$

where $\mathscr{P}_{\varepsilon}(\xi)=\lambda|\xi|^{4}-\frac{1}{4}\left(m^{2}+\delta m^{2}\right)|\xi|^{2}$ is related to the unit lattice action (1.1) by

$$
S_{\varepsilon}=S\left(\mathscr{S}_{\varepsilon} \phi, \mathscr{S}_{\varepsilon} u\right)
$$




\section{The First Renormalization Step}

The renormalization transformation $R$ defines a mapping

$$
R: S \rightarrow S^{(1)},
$$

where $S, S^{(1)}$ are both unit lattice actions. This transformation involves two steps:

(i) Integrate high momentum degrees of freedom while fixing block spin averages.

(ii) Rescale the resulting $L$-lattice action to the unit lattice.

Thus we can write

$$
R=\mathscr{S}_{L} \mathscr{T}
$$

where $\mathscr{T}$ is defined by

$$
\left(\mathscr{T} e^{-S}\right)(v, \psi)=\int e^{-S(u, \phi)} \delta_{A x}(u) \delta(v / Q u) \delta_{H}(\psi-Q \phi) \mathscr{D} u \mathscr{D} \phi .
$$

In (3.3) we specify the axial gauge with $\delta_{A x}(u)$ which sets $u_{b}=1$ on the set of bonds chosen as follows: Within each $L$-block $B(y)$ with corner $y$ in the $L$-lattice, let $T(y)$ denote the tree composed of unit bonds in $\Gamma_{y x}$. Here $x$ ranges over $B(y)$, and $T(y)$ is maximal - in the sense that adjoining any additional unit bond $b$ in $B(y)$ to $T(y)$ makes $T(y)$ multiply connected. Then

$$
\delta_{A x}(u)=\prod_{y \in T_{L}} \prod_{b \in T(y)} \delta\left(u_{b}\right) .
$$

Let $\mathscr{G}_{0}$ denote the subgroup of gauge transformations which are specified by $h$ 's in (2.7) which are constant on each $L$-block $B(y)$. These gauge transformations preserve the axial gauge and affect only bonds on the unit lattice which connect different blocks. They generate the gauge group of the integral $\mathscr{T}(\exp -S)$.

The factor $\delta(v / Q u)$ is a delta function which specifies that the average gauge field in each block $B(y)$ is $v$. Thus for $b^{\prime}$ an $L$-lattice bond,

$$
\delta(v / Q u)=\prod_{b^{\prime} \in T_{L}} \delta\left(v_{b^{\prime}} /(Q u)_{b^{\prime}}\right) .
$$

The factor $\delta_{H}$ is an approximate delta function which specifies that the block averages of the scalar (Higgs) field equal $\psi$. In particular, if $y$ denotes an $L$-lattice site,

$$
\delta_{H}(\psi-Q \phi)=\prod_{y \in T_{L}}\left[(a / 2 \pi) \exp \left(-(1 / 2) a|\psi-Q(u) \phi|^{2}\right)\right] .
$$

The integral (3.2) therefore has the normalization property

$$
\int \mathscr{T} e^{-S} \mathscr{D} v \mathscr{D} \psi=\int e^{-S} \mathscr{D} u \mathscr{D} \phi,
$$

where $\mathscr{D} v \mathscr{D} \psi$ is a measure corresponding to (3.3) but on the $L$-lattice.

Having defined the renormalization transformation, we now analyze its behavior for small fields. This will yield a Gaussian approximation to the integral (3.1) and a corresponding quadratic form for $S^{(1)}$. The quadratic form has the structure

$$
S_{Q}^{(1)}=\frac{1}{2}\left\langle f^{(1)}, \sigma_{1} f^{(1)}\right\rangle+\frac{1}{2}\left\langle\psi, \Delta_{1}\left(u_{1}\right) \psi\right\rangle,
$$

which we now derive and analyze. In particular we will establish strict positivity of $\sigma_{1}$ and $\Delta_{1}\left(u_{1}\right)$ in (3.8). 
We begin the study of (3.1) by rewriting the Wilson action contribution to $S(u, \phi)$. First define a unit lattice field $u_{b}^{\prime}$ with block averages equal to 1 . In this way we can write the resulting $u_{b}^{\prime}$ in terms of a vector potential which fluctuates about zero. Let

$$
\left(u^{\prime}\right)_{b}=\left\{\begin{array}{lll}
u_{b}, & \text { if } & b \notin B^{s}(b) \\
u_{b} v_{b^{\prime}}^{-1}, & \text { if } & b \in B^{s}\left(b^{\prime}\right)
\end{array}\right.
$$

be given in terms of the averaging operator $Q$ of $(2.10)$, where

$$
v_{b^{\prime}}=(Q u)_{b^{\prime}} .
$$

It then follows that

$$
\left(Q u^{\prime}\right)_{b^{\prime}}=1
$$

for all $L$-lattice bonds $b^{\prime}$. Now define $A_{b}^{\prime}$ by

$$
\left(u^{\prime}\right)_{b}=\exp \left(i e(\varepsilon) A_{b}^{\prime}\right) \text {. }
$$

Then the Wilson action can be written

$$
\sum_{p \in T_{1}} e(\varepsilon)^{-2}(1-\operatorname{Re} u(p))=\frac{1}{2} \sum_{p \in T_{1}}\left|\partial A^{\prime}+L^{-d / 2} Q^{e *} F\right|^{2}+O\left(e(\varepsilon)^{2}\right) .
$$

Here we define the $L$-lattice field $F\left(p^{\prime}\right)$ in terms of $v\left(p^{\prime}\right)$, where

$$
v\left(p^{\prime}\right) \equiv \prod_{b^{\prime} \in \partial p^{\prime}} v_{b^{\prime}} \equiv \exp \left[i e(L \varepsilon) F\left(p^{\prime}\right)\right] .
$$

The factor $L^{-d / 2}$ in (3.13) then arises from combining the factor $L^{-2}$ from (2.22) with $L^{-(d-4) / 2}=e(\varepsilon) / e(L \varepsilon)$.

The quadratic form in (3.13) can be reexpressed by translating to its minimum. By the Appendix, with $\alpha=\partial$ and $B=L^{-d / 2} Q^{e *} F$, we have

$$
A_{c l}=-L^{-d / 2} C \partial^{*} Q^{e *} F \text {. }
$$

Here $C=\left(\partial^{*} \partial\right)^{-1}$, restricted to the subspace of $A$ satisfying both $Q A=0$ and the axial gauge condition. Then by Corollary $\mathrm{A} 2$, and

$$
\begin{gathered}
A^{\prime}=A+A_{c l}, \\
\frac{1}{2} \sum_{p \in T_{\mathfrak{t}}}\left|\partial A^{\prime}+L^{-d / 2} Q^{e *} F\right|^{2}=\frac{1}{2} \sum_{p \in T_{\mathfrak{t}}}|\partial A|^{2}+\frac{1}{2}\left\langle F, \sum F\right\rangle,
\end{gathered}
$$

where

$$
\Sigma=L^{-d} Q^{e}\left(I-\partial C \partial^{*}\right) Q^{e *} .
$$

The fluctuation field $A$ lives on the unit lattice, and will be integrated. For small fluctuations we use this Gaussian approximation to yield a perturbative expression for the integral. Large fluctuations will lead to small probabilities.

The quadratic form $\left\langle F, \sum F\right\rangle$ provides the formula for the background field and it will come outside the integral, since the average fields $v$ and hence $F$ are fixed. This form will be scaled to the unit lattice. This scaling absorbs the factor $L^{-d}$, so

$$
\mathscr{S}_{L}\left\langle F, \sum F\right\rangle=\left\langle f^{(1)}, \sigma_{1} f^{(1)}\right\rangle,
$$


where

$$
\sigma_{1}=Q^{e}\left(I-\partial C \partial^{*}\right) Q^{e *}, \quad f^{(1)}(p)=\frac{1}{i e(L \varepsilon)} \ln v(p) .
$$

Here $Q^{e *}$ maps the unit lattice to the $L^{-1}$ lattice, and $\partial C \partial^{*}$ is an $L^{-1}$ lattice operator.

Define the unit lattice field

$$
u^{(1)}=u \exp (-i e(\varepsilon) A),
$$

with $A$ the fluctuation field defined by (3.16). Then after scaling back to the $L^{-1}$ lattice,

$$
u_{1} \equiv \mathscr{S}_{L}^{*} u^{(1)}
$$

is the gauge field on the $L^{-1}$ lattice on which the unit lattice renormalized action depends.

Let us now direct attention to the boson part of the integral (3.1). We expand

$$
S(u, \phi)+\frac{1}{2} a\|\psi-Q(u) \phi\|^{2}
$$

to identify the quadratic terms in $\phi$. Using (3.23), we also expand $u$ about its background value $u_{1}$, yielding additional contributions from $A$ which are accompanied by (small) factors $O(e(\varepsilon)$ ). Thus the leading terms in the Higgs action are

$$
\frac{1}{2} a\left\|Q\left(u^{(1)}\right) \phi-\psi\right\|^{2}+\frac{1}{2}\left\langle\phi, A_{u^{(1)}} \phi\right\rangle,
$$

with all corrections included in the potential $V_{1}\left(A, \phi, f_{1}\right)$ which have coefficients $O(\varepsilon)$.

Define

$$
G=\left(-\Delta_{u^{(1)}}+a Q\left(u^{(1)}\right)^{*} Q\left(u^{(1)}\right)\right)^{-1} .
$$

The quadratic form $(3,25)$ is minimized by

$$
\phi_{c l}=-a G Q\left(u^{\top}\right)^{*} \psi .
$$

See Appendix A. Writing $\phi=\phi_{c l}+\phi_{f l}$, expand (3.24) into background and fluctuation parts, yielding

$$
\frac{1}{2}\left\langle\phi_{f l}, G^{-1} \phi_{f l}\right\rangle+\frac{1}{2} a\left\langle\psi,\left[I-a Q G Q^{*}\right] \psi\right\rangle .
$$

In (3.28) the field $\phi_{f l}$ is a unit lattice field but $\psi$ is an $L$-lattice field. The scaling to the unit lattice will rewrite the second term in (3.28) in terms of a unit lattice field $\psi^{(1)}=\mathscr{S}_{L}^{*} \psi$. Let $G_{1}=\mathscr{S}_{L}^{*} G \mathscr{S}_{L}=G_{1}\left(u_{1}\right)$ denote the scaled Green's function, which is expressed in terms of the $L^{-1}$ lattice gauge field $u_{1}$.

\section{Form of the $\boldsymbol{k}^{\text {th }}$ Effective Action}

In this section we state the quadratic or mean field approximation to the action which is produced after $k$ renormalization transformations. We define the basic objects and give some detailed formulas for the quadratic action. Basically the 
action is the sum of two parts: a gauge field action and a scalar field part which is quadratic in $\phi$ and depends on an average value of the gauge field. There are further interaction terms between the gauge field and the scalar field, but these interactions yield small corrections to our mean field action and are not considered here.

We begin with a discussion of the gauge field terms. The scalar field is more straightforward and is postponed to the final subsection. The formulas which we give here are justified in Sect. 6, when we show that renormalization of the $k^{\text {th }}$ effective action yields the quadratic form for the $(k+1)^{s t}$ effective action. The action has the form

$$
S_{k}(v, \psi)=\frac{1}{2}\left\langle f^{(k)}, \sigma_{k} f^{(k)}\right\rangle+\frac{1}{2}\left\langle\psi, \Delta_{k}\left(u_{k}\right) \psi\right\rangle+\text { interaction terms, }
$$

where the interaction terms will be considered in detail in a later paper.

\subsection{The Axial Gauge Propagator and Minimizer}

We require an axial gauge propagator to express the effective action. Define $G_{k, A x}$ by the functional integral

$$
\exp \left(\frac{1}{2}\left\langle J, G_{k, A x} J\right\rangle\right)=Z_{k, A x}^{-1} \int \mathscr{D} A \delta\left(Q_{k} A\right) \delta_{k, A x}(A) \exp \left(-\frac{1}{2}\|\partial A\|^{2}+\langle A, J\rangle\right) .
$$

Here the normalization factor $Z_{k, A x}$ can be computed by setting $J=0$. The averaging operator $Q_{k}$ is the $k$-fold composition of the 1-step averaging operators $Q$ for bond variables, $Q_{k}=(Q)^{k}$. It follows that $Q_{k}$ is given by the formula (2.13), where $L$ is replaced by $L^{k}$. Finally, the axial gauge is fixed by the delta function

$$
\delta_{k, A x}(A) \equiv \prod_{j=0}^{k-1} \delta_{A x}\left(Q_{j} A\right) .
$$

The $j^{\text {th }}$ factor in the product acts on the $L^{j} \eta=L^{j-k}$ lattice. It sets bond field averages $Q_{j} A$ to zero on contours $\Gamma_{y x}$ defined in the $L^{j} \eta$ lattice in the same way that the axial gauge was fixed in Sect. 3 on contours in the unit lattice.

The reader may wonder whether $\partial$ has zero modes on the subspace of gauge fields satisfying $Q_{k} A=0$ and satisfying the axial gauge condition. Such zero modes do not occur, and as a consequence the integral (4.1.1) is convergent also for noncompact gauge fields. A proof of this fact for $k=1$ follows by considering on each plaquette in a lattice block the condition $\partial A=0$ and the axial gauge condition. This shows that $A$ can be nonzero only on bonds connecting different blocks, and it must be constant on the bonds connecting two given blocks. The condition $Q A=0$ then ensures that $A$ is everywhere zero. Similar, elementary reasoning yields an inductive proof for $k>1$, but we leave out the details.

The propagator $G_{k, A x}$ itself is the second moment of the measure $\exp \left(-\frac{1}{2}\|\partial A\|^{2}\right)$, restricted to the space of gauge fields which satisfy the $k^{\text {th }}$ axial gauge condition and condition on the $k^{\text {th }}$ average fields vanishing. We do not know a simple operator theoretic definition of $G_{k, A x}$, and we generally establish the properties of the propagator by appealing to the definition (4.1.1).

Another aspect of the action $S_{G}(A)=\frac{1}{2}\|\partial A\|^{2}$ concerns what configurations minimize $S_{G}(A)$ subject to the constraints of a gauge condition and a condition that the average field $Q_{k} A$ equals a given value $B$. We introduce a transformation 
$H_{k, A x}$ which maps $B$ into such a minimizing configuration for axial gauge, and we call $H_{k, A x}$ the axial gauge minimizer. Explicitly,

$$
H_{k, A x} B=Z_{k, A x}(B)^{-1} \int \mathscr{D} A \delta\left(Q_{k} A-B\right) \delta_{k, A x}(A) A \exp \left(-\frac{1}{2}\|\partial A\|^{2}\right),
$$

where

$$
Z_{k, A x}(B)=\int \mathscr{D} A \delta\left(Q_{k} A-B\right) \delta_{k, A x}(A) \exp \left(-\frac{1}{2}\|\partial A\|^{2}\right) .
$$

Note that by definition

$$
Q_{k} H_{k, A x} B=B .
$$

\subsection{The Quadratic Action and Plaquette Fields}

We begin with the definition of the basic quadratic form $\sigma_{k}$. Again we use a functional integral definition

$$
\exp \left(-\frac{1}{2}\left\langle f, \sigma_{k} f\right\rangle\right)=Z_{k, A x}^{-1} \int \mathscr{D} A \delta\left(Q_{k} A\right) \delta_{k, A x}(A) \exp \left(-\frac{1}{2}\left\|\partial A-Q_{k}^{e *} f\right\|^{2}\right) .
$$

Here $f$ is any real (Lie algebra) valued field defined on unit lattice plaquettes.

Clearly $\sigma_{k}$ is related to the propagator $G_{k, A x}$ of Sect. 4.1. Comparing (4.2.1) with (4.1.1) we find

$$
\sigma_{k}=Q_{k}^{e}\left(I-\partial G_{k, A x} \partial^{*}\right) Q_{k}^{e *}=\eta^{-2} I-Q_{k}^{e} \partial G_{k, A x} \partial^{*} Q_{k}^{e *} .
$$

Here we have used $Q_{k}^{e} Q_{k}^{e *}=\eta^{-2}$, see (2.24). Note that if $k=1$, then $G_{k, A x}=C$ and (4.2.2) agrees with (3.21).

The right side of (4.2.2) involves the combination $\partial G_{k, A x} \partial^{*}$ which is invariant under a change of gauge. Hence the gauge chosen to define $\sigma_{k}$ is irrelevant, and the quadratic form $\sigma_{k}$ is gauge invariant. We use this property in Sect. 7 when we derive an explicit momentum-space representation for $\sigma_{k}$. Using that representation we also establish a uniform, positive lower bound

$$
0<c \leqq \sigma_{k} .
$$

The quadratic part of the effective action is obtained by evaluating $\sigma_{k}$ on a special field configuration $f^{(k)}$ related to the gauge field after $k$ renormalization steps. Let $v$ be defined for unit lattice bonds, with values in the group U(1). Define the plaquette field $f^{(k)}$ on the unit lattice by

$$
f^{(k)}=\left(i e_{k}\right)^{-1} \ln v(\partial p), \quad p \in T_{1}^{(k)}, \text { where } e_{k}=e\left(L^{k} \varepsilon\right)^{(4-d) / 2} .
$$

The quadratic part of the action for the gauge field is then

$$
\frac{1}{2}\left\langle f^{(k)}, \sigma_{k} f^{(k)}\right\rangle \text {. }
$$

We also use a field $f_{k}$ which lives on the $\eta=L^{-k}$ lattice and is defined by

$$
f_{k}=\left(I-\partial G_{k, A x} \partial^{*}\right) Q_{k}^{e *} f^{(k)} \text {. }
$$

Thus $f_{k}(p)$ is a function with $p \in T_{\eta}$. The action (4.2.5) also can be written

$$
\frac{1}{2}\left\langle f^{(k)}, \sigma_{k} f^{(k)}\right\rangle=\frac{1}{2}\left\|f_{k}\right\|^{2} \text {. }
$$


This identity is a consequence of the fact that $\partial G_{k, A x} \partial^{*}$ is a projection operator. Alternatively, we compute from (4.1.1) and (4.2.1) that

$$
\exp \left(-\frac{1}{2}\left\langle f, \sigma_{k} f\right\rangle\right)=\exp \left(\frac{1}{2}\left\langle\partial^{*} Q_{k}^{e *} f, G_{k, A x} \partial^{*} Q_{k}^{e *} f\right\rangle\right) \exp \left(-\frac{1}{2}\left\|Q_{k}^{e *} f\right\|^{2}\right),
$$

from which (4.2.7) follows.

\subsection{Quadratic Action for Curls}

The quadratic form $\sigma_{k}$ simplifies on curls, namely for fields $f$ of the form $f=\partial B$. In particular

$$
\left\langle\partial B, \sigma_{k} \partial B\right\rangle=\left\langle B, \Delta_{k} B\right\rangle,
$$

which defines an action $A_{k}$. To give a functional integral representation for $\Delta_{k}$, we note that

$$
\begin{aligned}
\exp \left(-\frac{1}{2}\left\langle\partial B, \sigma_{k} \partial B\right\rangle\right) & =Z_{k, A x}^{-1} \int \mathscr{D} A \delta\left(Q_{k} A\right) \delta_{k, A x}(A) \exp \left(-\frac{1}{2}\left\|\partial A-Q_{k}^{e *} \partial B\right\|^{2}\right) \\
& =Z_{k, A x}^{-1} \int \mathscr{D} A \delta\left(Q_{k} A-B\right) \delta_{k, A x}(A) \exp \left(-\frac{1}{2}\|\partial A\|^{2}\right) \\
& =\exp \left(-\frac{1}{2}\left\langle B, A_{k} B\right\rangle\right) .
\end{aligned}
$$

Here we have performed a translation of $A$ on surface bonds $A \rightarrow A+Q_{k}^{s *} B$. We use the fact that the axial gauge condition is independent of the surface bonds, we use $\partial Q_{k}^{s *}=Q_{k}^{e *} \partial$, and we also use (2.19).

The action $\Delta_{k}$ yields the unit lattice propagator $C^{(k)}$, defined as follows:

$$
\exp \left(\frac{1}{2}\left\langle J, C^{(k)} J\right\rangle\right)=\left(Z^{(k)}\right)^{-1} \int \mathscr{D} B \delta(Q B) \delta_{A x}(B) \exp \left(-\frac{1}{2}\left\langle B, A_{k} B\right\rangle+\langle B, J\rangle\right) \text {. }
$$

The actions $\Delta_{k}$ and propagators $C^{(k)}$ were studied by Balaban [6II] who established that $C^{(k)}$ is well defined and is bounded in norm

$$
\left\|C^{(k)}\right\| \leqq c
$$

uniformly in $k$. Furthermore $C^{(k)}$ has a kernel which decays exponentially, uniformly in $k$.

$$
\left|C^{(k)}(x, y)\right| \leqq a \exp (-b|x-y|) .
$$

\subsection{Landau Gauge Propagator and Minimizer}

The axial gauge has many useful properties, especially in providing convenient definitions of Green's functions, and for establishing many algebraic identities in Sect. 5. However, the axial gauge does not provide either good regularity or good decay properties for the Green's functions. These are much better in Landau gauge.

Landau gauge is defined for our lattice theory in a complicated way, because of the restrictions on gauge transformations. We introduce a gauge fixing function $\mathscr{G}\left(\partial^{*} A\right)$. The exact form of $\mathscr{G}$ is important for the proof of estimates. An appropriate choice is

$$
\mathscr{G}\left(\partial^{*} A\right)=\exp \left(-\frac{1}{2}\left\|\partial^{*} A\right\|^{2}\right) /\left(\int d \lambda \delta\left(Q^{\prime} \lambda\right) \exp \left(-\frac{1}{2}\left\|\partial^{*} A-\Delta \lambda\right\|^{2}\right)\right),
$$


where $Q^{\prime}$ denotes the ordinary average over $k$-blocks. Such a Faddeev-Popov type choice leads to the formula for the Landau gauge minimizer

$$
H_{k} B=Z_{k}(B)^{-1} \int \mathscr{D} A \delta\left(Q_{k} A-B\right) \mathscr{G}\left(\partial^{*} A\right) A \exp \left(-\frac{1}{2}\|\partial A\|^{2}\right),
$$

where

$$
Z_{k}(B)=\int \mathscr{D} A \delta\left(Q_{k} A-B\right) \mathscr{G}\left(\partial^{*} A\right) \exp \left(-\frac{1}{2}\|\partial A\|^{2}\right) .
$$

We could define a well-behaved, Landau gauge propagator $G_{k}$ by a formula similar to (4.4.1), but with $\mathscr{G}\left(\partial^{*} A\right)$ replacing $\delta_{k, A x}(A)$. However, we do not use this propagator in our consideration of the Higgs effect. Rather, we define a propagator $\mathscr{D}_{k}$ in terms of minimizers $H_{j}$ and the covariance operators $C^{(j)}$ of Sect. 4.3. Let

$$
\mathscr{D}_{k} \equiv \sum_{j=0}^{k-1} H_{j} C^{(j)} H_{j}^{*} \text {. }
$$

Here the transformations $H_{j}$ are now defined by the same formulas as (4.4.2); however, they act on the $\eta=L^{-k}$ lattice instead of the $L^{-j}$ lattice and they involve $j^{\text {th }}$ order averages $Q_{j}$. Similarly the propagators $C^{(j)}$ are defined by the formula $(4.3 .3)$ but on the $L^{j} \eta=L^{j^{-k}}$ lattice, rather than on the unit lattice. They still involve onestep averages $Q$.

We remark that $\mathscr{D}_{k}, G_{k, A x}$, and $G_{k}$ are related by change of gauge formulas, some of which we establish in Sect. 5 .

\subsection{Bond Fields}

The first renormalization step in Sect. 3 yielded an effective action $S^{(1)}(v, \psi)$ depending on a unit lattice field $v$, through the $L^{-1}$ lattice field $u_{1}$. We need to generalize this picture to the $k$-step action $S^{(k)}$. After $k$ renormalization steps we again have as independent variables the gauge field $v$ defined on the unit lattice. We introduce a generalization of the configuration $u_{1}$ denoted $u_{k}$, To begin, we have to define a transformation $Q_{k}^{s *}$ from unit-lattice, group-valued configurations $v$ to $\eta$-lattice, group-valued configurations.

We can represent an arbitrary $v$ as

$$
v_{b}=\exp \left(i e_{k} B_{b}\right)
$$

where $B_{b}=\left(i e_{k}\right)^{-1} \ln v_{b}$, and the branch of the logarithm was chosen in (2.11). Then we extend the definition $Q_{s}^{*}$ from the Lie algebra to group variables $v$ by the definition

$$
\left(Q_{k}^{s *} v\right)_{b}=\exp \left(\left(i e_{k} \eta Q_{k}^{s *} B\right)_{b}\right), \quad b \in T_{\eta} .
$$

This raises the question of what happens if we choose another branch of the logarithm. The independence of the resulting transformation on this choice follows from the equivalent definition:

$$
\left(Q_{k}^{s *} v\right)_{b}= \begin{cases}1 & \begin{array}{l}
\text { if } b \text { is strictly contained in a } k \text {-block } \\
\text { (both endpoints belong to the block) }
\end{array} \\
v_{c} & \begin{array}{l}
\text { if the } \eta \text {-lattice bond } b \text { belongs to the corridor } \\
\text { of bonds connecting the two blocks } B^{k}\left(c_{-}\right) \text {and }
\end{array} \\
B^{k}\left(c_{+}\right) . \text {Here } c \text { is a unit lattice bond }\end{cases}
$$


Now we define

$$
\left(u_{k}\right)_{b}=\left(Q_{k}^{s * v}\right)_{b} \exp \left[-i e_{k} \eta\left(\mathscr{D}_{k} \partial^{*} Q_{k}^{e *} f^{(k)}\right)_{b}\right] .
$$

This field configuration $u_{k}$ is the minimal configuration for the approximate action, up to a gauge transformation. It serves as the background field (external gauge field) in the expressions for the scalar field action and propagators.

\subsection{The Scalar Field Action}

The fundamental propagator $G_{k}\left(u_{k}\right)$ for the scalar field in this theory can also be defined by a functional integral,

$$
\exp \left(\frac{1}{2}\left\langle h, G_{k}\left(u_{k}\right) h\right\rangle\right)=Z_{k}\left(u_{k}\right)^{-1} \int \mathscr{D} \phi \exp \left[-\frac{1}{2}\left\|D_{u_{k}} \phi\right\|^{2}-\frac{1}{2} a_{k}\left\|Q_{k}\left(u_{k}\right) \phi\right\|^{2}+\langle\phi, h\rangle\right] .
$$

Since no restrictions on $\phi$ occur in the Gaussian integral (4.6.1), we can also write

$$
G_{k}\left(u_{k}\right)=\left[-\Delta_{u_{k}}+a_{k} Q_{k}^{*}\left(u_{k}\right) Q_{k}\left(u_{k}\right)\right]^{-1},
$$

where

$$
-\Delta_{u_{k}}=D_{u_{k}}^{*} D_{u_{k}}
$$

The coefficients $a_{k}$ are produced by iterating one-step renormalization transformations which use a constant $a$ in the Gaussian. This yields $a_{k}=a\left(1-L^{-2}\right)\left(1-L^{-2 k}\right)^{-1}$ in the $k$-step transformation [3].

The quadratic form which arises for the scalar field is $\left\langle\psi, \Delta_{k}\left(u_{k}\right) \psi\right\rangle$, where $\psi$ is the unit lattice scalar field and where

$$
A_{k}\left(u_{k}\right)=a_{k} I-a_{k}^{2} Q_{k}\left(u_{k}\right) G_{k}\left(u_{k}\right) Q_{k}^{*}\left(u_{k}\right) .
$$

The scalar field action depends on the unit lattice field $\psi$ through the $\eta$-lattice minimizer $\psi_{k}$, where

$$
\psi_{k}=a_{k} G_{k}\left(u_{k}\right) Q_{k}^{*}\left(u_{k}\right) \psi
$$

The other propagators such as $C^{(k)}\left(u_{k}\right)$ are defined as in [3] with the only change that the background gauge field is $u_{k}$.

\section{Relations Among Minimizers and Propagators}

\subsection{Change of Gauge for Minimizers}

In this section we discuss relations among the operators $H$ and $G$. We begin by relating $H_{k, A x}$ and $H_{k}$. Recall that $H_{k} B$ is the configuration which minimizes the action $\|\partial A\|^{2}$, subject to a gauge condition as well as the restriction $Q_{k} A=B$ on field averages. We claim that $H_{k, A x} B$ and $H_{k} B$ differ by a gauge transformation,

$$
H_{k, A x} B-H_{k} B=\partial \lambda \text {. }
$$

We show that $\lambda$ is an explicit, linear function of $H_{k} B$. In order to specify $\lambda$, we choose a sequence of points $x_{0}, \ldots, x_{k}$, where $x=x_{0}, y=x_{k}$, and where

$$
x \in B^{j}\left(x_{j}\right), \quad x_{j} \in T_{L \eta \eta}^{(j)},
$$


that is

$$
x_{j} \in B\left(x_{j+1}\right)
$$

The contour $\Gamma_{x_{j}, x}$ runs from $x$ to $x_{1}$ in $B\left(x_{1}\right)$, from $x_{1}$ to $x_{2}$ in $B\left(x_{2}\right)$, etc.

Proposition 5.1.1. The relation (5.1.1) holds with

$$
\lambda(x)=-\sum_{j=0}^{k-1} L^{j-k}\left[\left(Q_{j} H_{k} B\right)\left(\Gamma_{x_{j+1}, x_{j}}\right)-\sum_{x^{\prime} \in B\left(x_{j}+1\right)}\left(L^{-d} Q_{j} H_{k} B\right)\left(\Gamma_{x_{j+1}, x^{\prime}}\right)\right] .
$$

Here we use $\left(Q_{j} H_{k} B\right)(\Gamma)=\sum_{b \in \Gamma}\left(Q_{j} H_{k} B\right)(b)$, with no factor of the lattice spacing for $\Gamma$.

Proof. The definition of $H_{k, A x} B$ is

$$
H_{k, A x} B=Z_{k, A x}(B)^{-1} \int d A \delta\left(Q_{k} A-B\right) \delta_{k, A x}(A) \exp \left(-\frac{1}{2}\|\partial A\|^{2}\right) A,
$$

where

$$
\delta_{k, A x}(A)=\prod_{j=0}^{k-1} \delta_{A x}\left(Q_{j} A\right)
$$

Furthermore,

$$
H_{k} B=Z_{k}(B)^{-1} \int d A \delta\left(Q_{k} A-B\right) \mathscr{G}\left(\partial^{*} A\right) \exp \left(-\frac{1}{2}\|\partial A\|^{2}\right) A,
$$

where $\mathscr{G}\left(\partial^{*} A\right)$ is a gauge fixing term, and where both integrals are normalized so that without the final factor $A$ they equal 1. We use a variant of the Faddeev-Popov procedure and insert the factor 1 in the integrand (5.1.5), expressed as

$$
1=Z_{k}^{-1} \int d \lambda \delta\left(Q_{k}^{\prime} \lambda\right) \mathscr{G}\left(\partial^{*} A-\Delta \lambda\right)
$$

Therefore,

$$
\begin{aligned}
Z_{k, A x}(B) & =\int d A \delta\left(Q_{k} A-B\right) \delta_{k, A x}(A) \exp \left(-\frac{1}{2}\|\partial A\|^{2}\right) \\
& =Z_{k}^{-1} \int d A d \lambda \delta\left(Q_{k}^{\prime} \lambda\right) \mathscr{G}\left(\partial^{*} A-\Delta \lambda\right) \delta\left(Q_{k} A-B\right) \delta_{k, A x}(A) \exp \left(-\frac{1}{2}\|\partial A\|^{2}\right) .
\end{aligned}
$$

Making the gauge transformation $A \rightarrow A+\partial \lambda$, and using $Q_{k} A \rightarrow Q_{k} A+Q_{k} \partial \lambda$ $=Q_{k} A+\partial Q_{k}^{\prime} \lambda=Q_{k} A$, on account of the $\delta$ function yields

$$
\begin{aligned}
Z_{k, A x}(B) & =Z_{k}^{-1} \int d A \mathscr{G}\left(\partial^{*} A\right) \delta\left(Q_{k} A-B\right) \exp \left(-\frac{1}{2}\|\partial A\|^{2}\right) \int d \lambda \delta\left(Q_{k}^{\prime} \lambda\right) \delta_{k, A x}(A+\partial \lambda) \\
& =Z_{k}^{-1} \int d A \mathscr{G}\left(\partial^{*} A\right) \delta\left(Q_{k} A-B\right) \exp \left(-\frac{1}{2}\|\partial A\|^{2}\right)=Z_{k}^{-1} Z_{k}(B)
\end{aligned}
$$

If we perform the same steps leading to (5.1.8) in (5.1.5), we obtain by (5.1.8)

$$
\begin{aligned}
H_{k, A x} B= & Z_{k}(B)^{-1} \int d A \delta\left(Q_{k} A-B\right) \mathscr{G}\left(\partial^{*} A\right) \exp \left(-\frac{1}{2}\|\partial A\|^{2}\right) \\
& \cdot \int d \lambda \delta\left(Q_{k}^{\prime} \lambda\right) \delta_{k, A x}(A+\partial \lambda)(A+\partial \lambda) .
\end{aligned}
$$

In order to evaluate (5.1.9), we solve the system of linear equations for $\lambda$ which result from the delta functions $\delta\left(Q_{k}^{\prime} \lambda\right)$ and $\delta_{k, A x}(A+\partial \lambda)$. Using this value of $\lambda=\lambda(A)$, we have

$$
H_{k, A x} B=Z_{k}(B)^{-1} \int d A \delta\left(Q_{k} A-B\right) \mathscr{G}\left(\partial^{*} A\right) \exp \left(-\frac{1}{2}\|\partial A\|^{2}\right)(A+\partial \lambda(A))
$$


We now study the equations which determine $\lambda(A)$. The axial gauge fixing conditions in (5.1.9) entails

$$
\left(Q_{j} A\right)\left(\Gamma_{z, z^{\prime}}\right)=-\left(L^{j} \eta\right)^{-1}\left[\left(Q_{j}^{\prime} \lambda\right)\left(z^{\prime}\right)-\left(Q_{j}^{\prime} \lambda\right)(z)\right],
$$

where $z$ and $z^{\prime}$ are endpoints of a bond in $B\left(x_{j+1}\right)$ which lies on an axial gauge fixing contour. Since every point in $B\left(x_{j+1}\right)$ lies on some such contour, we can sum these identities to show that for all $x^{\prime} \in B\left(x_{j+1}\right)$,

$$
\left(Q_{j} A\right)\left(\Gamma_{x_{j+1}, x^{\prime}}\right)=-\left(L^{j} \eta\right)^{-1}\left[\left(Q_{j}^{\prime} \lambda\right)\left(x^{\prime}\right)-\left(Q_{j}^{\prime} \lambda\right)\left(x_{j+1}\right)\right] .
$$

Summing over $x^{\prime} \in B\left(x_{j+1}\right)$ yields

$$
\left(Q_{j}^{\prime} \lambda\right)\left(x_{j+1}\right)=\left(Q_{j+1}^{\prime} \lambda\right)\left(x_{j+1}\right)+L^{j} \eta Q^{\prime}\left(Q_{j} A\right)\left(\Gamma_{x_{j+1}}, \cdot\right) .
$$

We use the fact that $\left(Q_{j}^{\prime} \lambda\right)\left(x_{j+1}\right)$ is constant in this sum, and the final $Q^{\prime}$ acts on ".". Substituting (5.1.11) in (5.1.10) with $x^{\prime}=x_{j}$ then gives

$$
\left(Q_{j}^{\prime} \lambda\right)\left(x_{j}\right)=\left(Q_{j+1}^{\prime} \lambda\right)\left(x_{j+1}\right)-L^{j} \eta\left[\left(Q_{j} A\right)\left(\Gamma_{x_{j+1}, x_{j}}\right)-Q^{\prime}\left(Q_{j} A\right)\left(\Gamma_{x_{j+1},}\right)\right] \text {. }
$$

The identities (5.1.12) hold for $j=0,1, \ldots, k-1$. Setting $j=0$ we have

$$
\lambda(x)=\left(Q_{0}^{\prime} \lambda\right)\left(x_{0}\right)=\left(Q^{\prime} \lambda\right)\left(x_{1}\right)-\eta\left[A\left(\Gamma_{x_{1}, x}\right)-\left(Q^{\prime} A\right)\left(\Gamma_{x_{1}}, \cdot\right)\right] .
$$

Furthermore, summing (5.1.12) from $j=0$ to $j=k-1$, the factors $\left(Q_{j}^{\prime} \lambda\right)\left(x_{j}\right)$ cancel except for $j=0$ and $j=k$. Thus

$$
\lambda(x)=\left(Q_{k}^{\prime} \lambda\right)\left(x_{k}\right)-\sum_{j=0}^{k-1} L^{j} \eta\left[\left(Q_{j} A\right)\left(\Gamma_{x_{j+1}, x_{j}}\right)-Q^{\prime}\left(Q_{j} A\right)\left(\Gamma_{x_{j+1},}\right)\right] .
$$

Using the $\delta$ function for $Q_{k}^{\prime} \lambda$, and $\eta=L^{-k}$ gives

$$
\lambda(x)=\lambda(A, x)=-\sum_{j=0}^{k-1} L^{j-k}\left[\left(Q_{j} A\right)\left(\Gamma_{x_{j+1}, x_{j}}\right)-Q^{\prime}\left(Q_{j} A\right)\left(\Gamma_{x_{j+1},}\right)\right] .
$$

We now notice that $\lambda=\lambda(A)$ is a linear function of $A$. Thus we can write $\lambda(A, x)=\lambda\left(H_{k} B, x\right)-\lambda\left(A-H_{k} B, x\right)$. The term $\lambda\left(H_{k} B, x\right)$ is independent of $A$ and comes outside the $A$ integral. Thus

$$
\begin{aligned}
H_{k, A x} B= & H_{k} B+\partial \lambda\left(H_{k} B\right)+Z_{k}(B)^{-1} \int d A \delta\left(Q_{k} A-B\right) \mathscr{G}\left(\partial^{*} A\right) \\
& \cdot \exp \left(-\frac{1}{2}\|\partial A\|^{2}\right) \partial \lambda\left(A-H_{k} B\right) .
\end{aligned}
$$

Since $H_{k} B$ minimizes the quadratic form $\|\partial A\|^{2}$ subject to the restrictions imposed by $\delta\left(Q_{k} A-B\right)$ and $\mathscr{G}$, the term in (5.1.14) involving $\partial \lambda\left(A-H_{k} B\right)$ is linear in $A-H_{k} B$ and hence its integral vanishes. Thus $H_{k, A x} B=H_{k} B+\partial \lambda\left(H_{k} B\right)$, and the proof of Proposition 5.1.1 is complete.

\subsection{Decomposition of Axial Gauge Green's Functions}

Let $C^{(k)}$ denote the unit lattice propagator defined in (4.3.3). Here we give a simple relation between $C^{(k)}$ and the axial gauge propagator $G_{k, A x}$ of (4.1.1). This relation is similar to the definition (4.4.4) of the Landau gauge propagator $\mathscr{D}_{k}$ in terms of the $C^{(j)}$. The algebraic identity for $G_{k, A x}$ will be very useful, especially in computing the form of the action after renormalization, even though the axial gauge propagator has poor regularity and decay properties. 
Proposition 6.2. The $G_{k, A x}$ satisfy the recursion relation

$$
G_{k, A x}=H_{k-1, A x} C^{(k-1)} H_{k-1, A x}^{*}+G_{k-1, A x},
$$

with the solution:

$$
G_{k, A x}=\sum_{j=0}^{k-1} H_{j, A x} C^{(j)} H_{j, A x}^{*} .
$$

Proof. Clearly (5.2.1) and (5.2.2) are equivalent. We prove (5.2.1) starting from the definition of $G_{k, A x}$, namely (4.1.1). Write $Q_{k}=Q Q_{k-1}$, and expand the integrand of (4.1.1) using

$$
\delta\left(Q_{k} A\right) \delta_{k, A x}(A)=\int \mathscr{D} B \delta(Q B) \delta\left(Q_{k-1} A-B\right) \delta_{A x}(B) \delta_{k-1, A x}(A) .
$$

Then translate the integrand with respect to $A$ to the minimum of the quadratic form $\frac{1}{2}\|\partial A\|^{2}-\langle A, J\rangle$, under the restriction $Q_{k-1} A=B$ and the appropriate axial gauge. The minimum is achieved at $H_{k-1, A x} B$, so we write

$$
A=A^{\prime}+H_{k-1, A x} B \text {. }
$$

Inserting this and using (4.1.5) gives

$$
\begin{aligned}
\exp \left(\frac{1}{2}\left\langle J, G_{k, A x} J\right\rangle\right)= & Z^{-1} \int \mathscr{D} B \delta(Q B) \delta_{A x}(B) \\
& \cdot \exp \left(-\frac{1}{2}\left\|\partial H_{k-1, A x} B\right\|^{2}+\left\langle B, H_{k-1, A x}^{*} J\right\rangle\right) \\
& \cdot \int \mathscr{D} A^{\prime} \delta\left(Q_{k-1} A^{\prime}\right) \delta_{k-1, A x}\left(A^{\prime}\right) \\
& \cdot \exp \left(-\frac{1}{2}\left\|\partial A^{\prime}\right\|^{2}+\left\langle A^{\prime}, J\right\rangle\right) .
\end{aligned}
$$

The $A^{\prime}$ integral yields $Z_{k-1, A x} \exp \left(\frac{1}{2}\left\langle J, G_{k-1, A x} J\right\rangle\right)$, while the $B$ integral gives

$$
Z Z_{k-1, A x}^{-1} \exp \left(\frac{1}{2}\left\langle H_{k-1, A x}^{*} J, C^{(k-1)} H_{k-1, A x}^{*} J\right\rangle\right) \text {. }
$$

Thus (5.2.2) holds and the proposition follows.

Now that we have related $H_{k}$ and $H_{k, A x}$, we can find the relation between $\mathscr{D}_{k}$ and $G_{k, A x}$. In fact we need only the compositions $\mathscr{D}_{k} \partial^{*}$ and $G_{k, A x} \partial^{*}$, since only these products occur.

Proposition 5.2.2. There is a gauge transformation $D$ such that

$$
G_{k, A x} \partial^{*}-\mathscr{D}_{k} \partial^{*}=\partial D
$$

Explicitly

$$
D=\sum_{j=0}^{k-1} \lambda_{j}\left(H_{j} C^{(j)} H_{j}^{*} \partial^{*}\right),
$$

where $\lambda_{j}$ is the function of Proposition 5.1.1 with $k$ set equal to $j$. Here we write the operator identity, rather than the identity $G_{k, A x} \partial^{*} B=\mathscr{D}_{k} \partial^{*} B+\partial D B$ for configurations.

Proof. We use three facts: the formula (5.2.1), the fact that

$$
H_{j, A x}^{*} \partial^{*}=H_{j}^{*} \partial^{*},
$$


is gauge invariant, and formulas (5.1.1), (5.1.4) for the factor $H_{j, A x}-H_{j}$ in the expression

$$
G_{k, A x} \partial^{*}-\mathscr{D}_{k} \partial^{*}=\sum_{j=0}^{k-1}\left(H_{j, A x}-H_{j}\right) C^{(j)} H_{j}^{*} \partial^{*} .
$$

Substitution then yields (5.2.7).

Remark 1. A consequence of the proposition is

$$
\partial G_{k, A x} \partial^{*}=\partial \mathscr{D}_{k} \partial^{*} .
$$

Remark 2 . The gauge field quadratic form $\left\langle f^{(k)}, \sigma_{k} f^{(k)}\right\rangle$ can now be written entirely as a function of the $\eta$-lattice gauge field $u_{k}$. In fact

$$
\left\langle f^{(k)}, \sigma_{k} f^{(k)}\right\rangle=\sum_{p \in T_{\eta}} \eta^{d}\left|f_{k}(p)\right|^{2},
$$

where

$$
f_{k}(p)=\left(i e_{k} \eta^{2}\right)^{-1} \ln u_{k}(\partial p) .
$$

This follows from the definition (4.5.4) of $u_{k}$, giving

$$
u_{k}(\partial p)=\exp \left[i e_{k} \eta^{2} Q_{k}^{e *} f^{(k)}-i e_{k} \eta^{2} \partial \mathscr{D}_{k} \partial^{*} Q_{k}^{e *} f^{(k)}\right] .
$$

From this and (5.2.10) we get

$$
\left(i e_{k} \eta^{2}\right)^{-1} \ln u_{k}(\partial p)=Q_{k}^{e *} f^{(k)}-\partial G_{k, A x} \partial^{*} Q_{k}^{e *} f^{(k)},
$$

coinciding with (4.2.6).

\subsection{Minimizers and Green's Functions}

To help define the fluctuation field in the $(k+1)^{\mathrm{st}}$ step, we need one more identity, namely

$$
H_{k, A x} B=Q_{k}^{s *} B-G_{k, A x} \partial^{*} Q_{k}^{e *} B .
$$

This identity follows by inspecting the definition (4.1.3) after the translation $A=A^{\prime}+Q_{k}^{s *} B$. Use the facts $Q_{k} Q_{k}^{s *}=I$ and $\partial Q_{k}^{s *} B=Q_{k}^{e *} \partial B$ to obtain

$H_{k, A x} B=Z_{k, A x}(B)^{-1} \int \mathscr{D} A^{\prime} \delta\left(Q_{k} A^{\prime}\right) \delta_{k, A x}\left(A^{\prime}\right)\left(A^{\prime}+Q_{k}^{s *} B\right) \exp \left(-\frac{1}{2}\left\|\partial A^{\prime}+Q_{k}^{e *} \partial B\right\|^{2}\right)$.

The second term yields $Q_{k}^{s *} B$. In the first term we translate to the minimum of the quadratic form which is $G_{k, A x} \hat{O}^{*} Q_{k}^{e *} \partial B$. We see this by integrating.

\section{Renormalization Transformations Produce the Effective Action as Claimed}

In this section we study the renormalization of the quadratic part of the $k^{\text {th }}$ action $S_{k}$ in (4.1). This action depends on the plaquette field $f^{(k)}$, the (background) bond field $u_{k}$ and the scalar field $\psi$. Under renormalization we prove that $S_{k} \rightarrow S_{k+1}$. We exhibit this transformation by writing $S_{k}$ as a sum of two terms: the first is $S_{k+1}$, but scaled from the unit lattice to the $L$-lattice. The second term is the contribution of the fluctuation field on the unit lattice. The fluctuation field will be integrated in 
performing the renormalization transformation, while the remaining terms scale back to give $S_{k+1}$ on the unit lattice. In (4.1) we ignore the small corrections due to interactions and concentrate on the quadratic terms. Hence the result of this section is to justify our (otherwise ad hoc) choice of $\sigma_{k}$ and $\Delta_{k}\left(u_{k}\right)$ as the mean field quadratic forms for the $k^{\text {th }}$ effective action.

In accord with the notation of Sect. 3 we denote the variables in $S_{k}$ as $u$ and $\phi$ which take their values on the unit lattice. Thus we study

$$
\int \mathscr{D} u \mathscr{D} \phi \delta(v / Q u) \delta_{A x}(u) \exp \left[-\frac{1}{2} a\left\|\psi-Q\left(u_{k}\right) \phi\right\|^{2}-S_{k}(u, \phi)\right] .
$$

In this integral we perform a preparatory translation (the first translation in Sect. 3) to isolate the average gauge field on the $k^{\text {th }}$ length scale. Let $v$ denote the average gauge field on the $L$-lattice, and on the unit lattice let

$$
u=u^{\prime} Q^{s *} v, \quad u^{\prime}=\exp \left(i e_{k} B^{\prime}\right) .
$$

The plaquette field

$$
f^{(k)}(p)=\left(i e_{k}\right)^{-1} \ln u(\partial p), \quad p \in T_{1},
$$

then decomposes as

$$
f^{(k)}(p)=\left(i e_{k}\right)^{-1} \ln u^{\prime}(\partial p)+\left(i e_{k}\right)^{-1} \ln \left(Q^{s *} v\right)(\partial p)=\left(\partial B^{\prime}\right)(p)+L^{-d / 2}\left(Q^{e * f^{(k+1)}}\right)(p),
$$

which is valid as long as $u^{\prime}(p)$ and $\left(Q_{k}^{s *} v\right)(p)$ are close to 1 . The new field $f^{(k+1)}$ is an $L$-lattice field, but $Q_{k}^{e *}$ maps it back (to edge plaquettes) in the unit lattice.

The integral (6.1) will be studied in detail in later papers by dividing it into small and large field regions, defined by some restrictions on the range of $\phi$ and $u$. In this paper we are concerned with the small field regions which give the dominant contributions to the integral. In this region the identity (6.4) holds.

We will also see that the perturbative terms in the action are unaffected by the restriction to small fields, since these restrictions become trivial in the limit of zero coupling constants. In particular, this means that in the calculation of leading terms in the action $S_{k+1}$ we can assume that the integrations over fields in (6.1) are restricted only by the delta functions which appear in the definition of the renormalization transformation.

\section{1. $\sigma_{k}=\sigma_{k+1}+$ Fluctuation Form}

The goal here is to show that

$$
\left\langle f^{(k)}, \sigma_{k} f^{(k)}\right\rangle=\mathscr{S}_{L}^{-1}\left\langle f^{(k+1)}, \sigma_{k+1} f^{(k+1)}\right\rangle+\left\langle B, A_{k} B\right\rangle .
$$

In other words, the quadratic form $\sigma_{k}$ in $S_{k}$ which occurs in the integrand (6.1) can be decomposed into the sum of two independent forms: the $(k+1)$-step quadratic form scaled to the $L$-lattice and the quadratic form for the fluctuation field $B$. Hence the fluctuation field can be integrated and, after rescaling to the unit lattice, we obtain the desired $(k+1)$-step gauge field quadratic form. Using (6.4),

$$
\begin{aligned}
\left\langle f^{(k)}, \sigma_{k} f^{(k)}\right\rangle= & \left\langle\partial B^{\prime}, \sigma_{k} \partial B^{\prime}\right\rangle+2 L^{-d / 2}\left\langle\partial B^{\prime}, \sigma_{k} Q^{e *} f^{(k+1)}\right\rangle \\
& +L^{-d}\left\langle f^{(k+1)}, Q^{e} \sigma_{k} Q^{e *} f^{(k+1)}\right\rangle .
\end{aligned}
$$


Use definition (4.3.1) of $\Delta_{k}$ and the representation (4.2.2) for $\sigma_{k}$. In addition, note that $Q^{e} Q_{k}^{e}=Q_{k+1}^{e}$. Then

$$
\begin{aligned}
\left\langle f^{(k)}, \sigma_{k} f^{(k)}\right\rangle= & \left\langle B^{\prime}, \Delta_{k} B^{\prime}\right\rangle+2 L^{-d / 2}\left\langle\left(I-\partial G_{k, A x} \partial^{*}\right) Q_{k}^{e *} \partial B^{\prime}, Q_{k+1}^{e *} f^{(k+1)}\right\rangle \\
& +L^{-d}\left\langle Q_{k+1}^{e *} f^{(k+1)},\left(I-\partial G_{k, A x} \partial^{*}\right) Q_{k+1}^{e *} f^{(k+1)}\right\rangle .
\end{aligned}
$$

By (5.3.1), $H_{k, A x}=Q_{k}^{s *}-G_{k, A x} \partial^{*} Q_{k}^{e *} \partial$. Apply $\partial$ to this identity, and use the gauge invariance statement

$$
\partial H_{k, A x}=\partial H_{k}
$$

and the identity $\partial Q_{k}^{s *}=Q_{k}^{e *} \partial$. It follows that

$$
\partial H_{k}=\left(I-\partial G_{k, A x} \partial^{*}\right) Q_{k}^{e *} \partial .
$$

Substituting (6.1.5) in (6.1.3) gives

$$
\begin{aligned}
\left\langle f^{(k)}, \sigma_{k} f^{(k)}\right\rangle= & \left\langle B^{\prime}, A_{k} B^{\prime}\right\rangle+2 L^{-d / 2}\left\langle B^{\prime}, H_{k}^{*} \partial^{*} Q_{k+1}^{e *} f^{(k+1)}\right\rangle \\
& +L^{-d}\left\langle Q_{k+1}^{e *} f^{(k+1)},\left(I-\partial G_{k, A x} \partial^{*}\right) Q_{k+1}^{e *} f^{(k+1)}\right\rangle .
\end{aligned}
$$

Consider the quadratic form (6.1.6) as a function of $B^{\prime}$ and subject to the axial gauge and averaging restrictions of the integral (6.1). The minimum of the form is given by Proposition A3 of the Appendix, namely

$$
B^{\prime}=-L^{-d / 2} C^{(k)} H_{k}^{*} \partial^{*} Q_{k+1}^{e *} f^{(k+1)} .
$$

The proposition is applicable with $\mathscr{H}_{0}$ the subspace of axial gauge configurations defined by the $k^{\text {th }}$ axial gauge delta function $\delta_{k, A x}\left(B^{\prime}\right)$ and the delta function $\delta\left(Q B^{\prime}\right)$. The covariance $C$ of the proposition then agrees with $C^{(k)}$ defined in (4.3.3), and this is also the covariance in (6.1.7).

Furthermore, the translation

$$
B^{\prime}=B-L^{-d / 2} C^{(k)} H_{k}^{*} \partial^{*} Q_{k+1}^{e *} f^{(k+1)}
$$

and the decomposition (A16) yield

$$
\begin{aligned}
& \left\langle f^{(k)}, \sigma_{k} f^{(k)}\right\rangle \\
& \quad=\left\langle B, \Delta_{k} B\right\rangle+L^{-d}\left\langle Q_{k+1}^{e *} f^{(k+1)},\left(I-\partial G_{k, A x} \partial^{*}-\partial H_{k} C^{(k)} H_{k}^{*} \partial^{*}\right) Q_{k+1}^{e *} f^{(k+1)}\right\rangle .
\end{aligned}
$$

Using (6.1.4) and the representation (5.1.15), we can rewrite (6.1.9) as

$$
\left\langle f^{(k)}, \sigma_{k} f^{(k)}\right\rangle=\left\langle B, A_{k} B\right\rangle+\mathscr{S}_{L}^{-1}\left\langle f^{(k+1)}, \sigma_{k+1} f^{(k+1)}\right\rangle,
$$

where the scaling $\mathscr{S}_{L}$ absorbs the factor $L^{-d}$ and where

$$
\sigma_{k+1}=Q_{k+1}^{e}\left(I-\partial G_{k+1, A x} \partial^{*}\right) Q_{k+1}^{e *} .
$$

This verifies the inductive hypothesis.

\section{2. $u_{k}=u_{k+1} \cdot$ Fluctuation (Modulo Gauge Transformation)}

We now investigate the effect of the two translations defined in Sect. 6.1 on the field $u_{k}$. This allows us to separate a fluctuation field and a gauge transformation which 
then leave $u_{k+1}$. Rewrite the definition of $u_{k}$ (with $u$ in place of $v$ ), so

$$
u_{k}=Q_{k}^{s *} u \exp \left(-i e_{k} \eta \mathscr{D}_{k} \partial^{*} Q_{k}^{e *} f^{(k)}\right) \text {. }
$$

By (6.2) we have

$$
Q_{k}^{s *} u=\left(Q_{k+1}^{s *} v\right) e^{i e_{k} \eta Q^{*} B^{\prime}}
$$

so substituting this and (6.3) into (6.2.1) we obtain

$$
u_{k}=Q_{k+1}^{s *} v \exp \left[i e_{k} \eta Q_{k}^{s *} B^{\prime}-i e_{k} \eta\left(\mathscr{D}_{k} \partial^{*} Q_{k}^{e *} \partial B^{\prime}\right)-i e_{k} \eta L^{-d / 2}\left(\mathscr{D}_{k} \partial^{*} Q_{k+1}^{e *} f^{(k+1)}\right)\right] .
$$

We have the identity $G_{k, A} \partial^{*}=\mathscr{D}_{k} \partial^{*}+\partial D$ to replace $\mathscr{D}_{k} \partial^{*}$ by $G_{k, A x} \partial^{*}$. Then we apply again the identity (5.3.1) to replace the first two terms in the exponential by $i e_{k} \eta H_{k, A x} B^{\prime}$. This takes care of the first translation.

The second translation (6.1.5) then yields

$$
\begin{aligned}
u_{k}= & Q_{k+1}^{s *} v \exp \left[i e_{k} \eta H_{k, A x} B-i e_{k} \eta L^{-d / 2} H_{k, A x} C^{(k)} H_{k}^{*} \partial^{*} Q_{k+1}^{e *} f^{(k+1)}\right. \\
& \left.-i e_{k} \eta L^{-d / 2}\left(\mathscr{D}_{k} \partial^{*} Q_{k+1}^{e *} f^{(k+1)}\right)\right] \cdot \text { (gauge trans). }
\end{aligned}
$$

We make another gauge transformation to replace the axial gauge minimizers by Landau gauge minimizers, and we combine the second and third term into an operator $\mathscr{D}_{k+1}$. After rescaling, the $L^{-d / 2}$ factor is absorbed and we have

$$
u_{k}=e^{i e_{k} \eta H_{k} B} u_{k+1} \text { (the gauge transformation), }
$$

where the gauge transformation is generated by

$$
\exp \left[i e_{k} D\left(Q_{k}^{e *} \partial B^{\prime}\right)+i e_{k} \lambda_{k}\left(H_{k} B\right)-i e_{k} L^{-d / 2} \lambda_{k}\left(H_{k} C^{(k)} H_{k} \partial^{*} Q_{k+1}^{e *} f^{(k+1)}\right)\right] .
$$

\subsection{Scalar Field Renormalization}

In Sect. 6.2 we established the general decomposition (6.2.5) for $u_{k}$,

$$
u_{k}=u_{k+1} e^{i e_{k} \eta H_{k} B} e^{i \eta \partial \omega},
$$

where $\omega$ denotes the gauge transformation (6.2.6). This gauge transformation does not influence the gauge field quadratic form $\sigma_{k}$, since this form is fully gauge invariant. More generally, expressions involving only the gauge field are gauge invariant, so we must discuss the scalar field part of the action.

In that case, the form $\Delta_{k}\left(u_{k}\right)$ does depend on the gauge transformation $\omega$. Our procedure for constructing $S_{k}$ has the general invariance

$$
S_{k}\left(u_{k} e^{-i \eta \hat{o} \lambda}, e^{i \lambda} \phi\right)=S_{k}^{\prime}\left(u_{k}, \phi\right)
$$

for a gauge transformation $\lambda$. While we do not prove this invariance here, it is clear in the case of the quadratic forms for which we write explicit formulas.

The scalar field integral has the form

$$
\int \mathscr{D} \phi \exp \left\{-\left[S_{k}\left(u_{k}, \phi\right)+\frac{1}{2} a\left\|\psi-Q\left(u_{k}\right) \phi\right\|^{2}\right]\right\} .
$$

If we apply the decomposition (6.3.1) for $u_{k}$, and the gauge invariance (6.3.2), then (6.3.3) becomes

$$
\int \mathscr{D} \phi \exp \left(-\left[S_{k}\left(u_{k+1} e^{i e_{k} \eta H_{k} B}, \phi\right)+\frac{1}{2} a\left\|e^{-i \omega} p-Q\left(u_{k+1} e^{i e_{k} \eta H_{k} B}\right) \phi\right\|^{2}\right]\right) .
$$


Here we use the gauge invariance of the measure $\mathscr{D} \phi$. The resulting integral still depends on $\omega$. However, the integral of (6.3.4) over $\psi$ is independent of $\omega$, since $\mathscr{D} \psi$ is also gauge invariant.

We can either write the new action in terms of $\psi^{\prime}=e^{-i \omega} \psi$, or use this gauge invariance and the fact that we are only interested in integrals of (6.3.4) over $\psi$, times other gauge invariant functions of $\psi$ (as observables). We take the latter point of view and ignore $\omega$.

Now we are in exactly the same situation as [3]. We expand the whole integrand with respect to $H_{k} B$ and perform the fluctuation field integral over boson fields as in that paper. It reproduces the $(k+1)$ quadratic form for the action.

\section{Positivity and Localization of the Effective Action}

Positivity bounds on $\sigma_{k}$ and $\Delta_{k}\left(u_{k}\right)$ are a necessary step in proving that the renormalization transformation preserves stability of the effective action. The main difficulty centers on the quadratic form $\sigma_{k}$, which arises from the gauge field action. In particular, $\sigma_{k}$ comes from the Wilson form of the action. Unlike the "Gaussian" action for the gauge field used in earlier studies of the U(1) Higgs model, the Wilson action has rather subtle positivity properties. We study this question first, yielding the main result of this section, $0<c \leqq \sigma_{k}$. We then finish by stating minor generalizations of known estimates which apply to the other operators $H_{k}, \mathscr{D}_{k}$, and $\Delta_{k}\left(u_{k}\right)$ introduced in this paper.

\subsection{Positivity of $\sigma_{k}$}

Theorem 7.1.1. There exists a constant $c>0$, independent of $k$, such that

$$
c \leqq \sigma_{k} .
$$

The strategy of the proof is to give an explicit formula for $\sigma_{k}$, which we then analyze in detail. Since we study periodic boundary conditions, $\sigma_{k}$ is translation invariant. Thus it is natural to study $\sigma_{k}$ as a multiplication operator $\sigma_{k}(p)$ in the Fourier transform representation. (Note that we now use $p$ to denote a momentum variable, rather than a plaquette variable.) The momenta $p$ have $d$ components $p_{i}$, and $\left|p_{i}\right| \leqq \pi$.

We introduce a tensor notation with functions $f_{\mu v}$ on plaquettes given as antisymmetric functions on coordinate axes $\mu, v$ which indicates plaquette orientation. They also depend on a lattice position. Then

$$
\left\langle f^{(k)}, \sigma_{k} f^{(k)}\right\rangle=\int_{-\pi}^{\pi} \ldots \int_{-\pi}^{\pi}\left\langle\tilde{f}^{(k)}(p), \sigma_{k}(p) \tilde{f}^{(k)}(p)\right\rangle d p,
$$

where the momentum $p$ inner product is defined by

$$
\left\langle\widetilde{f}(p), \sigma_{k}(p) \tilde{f}(p)\right\rangle=\sum_{\mu, \nu, \lambda, \kappa} \bar{f}_{\mu \nu}(p) \sigma_{k ; \mu \nu \lambda \kappa}(p) \tilde{f}_{\lambda \kappa}(p) .
$$

In order to study $\sigma_{k}(p)$ we need standard momentum space operations such as derivatives $\partial$ or Laplacians $\Delta$. Since we have two special lattice scales, the unit lattice leading to momenta $\left|p_{i}\right| \leqq \pi$ and the $\eta=L^{-k}$ lattice leading to momenta 
$\left|p_{i}\right| \leqq \pi / \eta=\pi L^{k}$, we require operators on both scales. We use a superscript (1) to denote the unit lattice scale.

With these conventions, the derivative $\partial$ can be represented by a diagonal $d \times d$ matrix $\partial(p)$ with eigenvalues

$$
\partial_{\mu}(p)=\left(\exp \left(i \eta p_{\mu}\right)-1\right) / \eta .
$$

The unit lattice derivative $\partial^{(1)}(p)$ is a similar matrix with eigenvalues

$$
\partial_{\mu}^{(1)}(p)=\exp \left(i p_{\mu}\right)-1 \text {. }
$$

The corresponding Laplacians are

$$
\Delta(p)=\operatorname{Tr} \partial(p)^{*} \partial(p), \quad \Delta^{(1)}(p)=\operatorname{Tr} \partial^{(1)}(p)^{*} \partial^{(1)}(p) .
$$

We require the operator $V(p)$ defined by

$$
V(p)=\partial^{(1)}\left(p^{\prime}\right) \partial(p)^{-1} \text {. }
$$

Here and below we use the notation $p_{i}^{\prime} \in[-\pi, \pi]$ and

$$
p^{\prime}=p \bmod 2 \pi
$$

so $p^{\prime}$ denotes a unit lattice momentum. The eigenvalues of $V(p)$ are $v_{\mu}(p)=\partial_{\mu}^{(1)}(p) / \partial_{\mu}(p)$. In terms of $V(p)$ we also define

and

$$
u(p)=\operatorname{det} V(p)
$$

$$
\varphi_{\mu}\left(p^{\prime}\right)=\sum_{\substack{\ell \in 2 \pi z^{a} \\\left|i_{i}\right| \geqq \pi / \eta}}\left|u\left(p^{\prime}+l\right) v_{\mu}\left(p^{\prime}+l\right)\right|^{2} \Delta\left(p^{\prime}+l\right)^{-1}
$$

In terms of these functions we can express the averaging operators $Q_{k}^{e}$, etc. For example

$$
\left(Q_{k}^{e} f\right)_{\mu v}^{\sim}\left(p^{\prime}\right)=\sum_{l} u\left(p^{\prime}+l\right) \tilde{f}_{\mu v}\left(p^{\prime}+l\right)\left(v_{\mu}\left(p^{\prime}+l\right) v_{v}\left(p^{\prime}+l\right)\right)^{-1} .
$$

The basic object we wish to study is $\sigma_{k}$, defined in (4.2.2),

$$
\sigma_{k}=Q_{k}^{e}\left(I-\hat{\partial} G_{k, A x} \partial^{*}\right) Q_{k}^{e *} \text {. }
$$

The axial gauge Green's function $G_{k, A x}$ has a very complicated momentum space structure. Thus we use gauge invariance of $\partial G_{k, A x} \partial^{*}$ to replace it by the Landau gauge operator $\partial G_{k} \hat{O}^{*}$. Here we emphasize that $G_{k}$ is a slightly different operator from $\mathscr{D}_{k}$ introduced in Sect. 4 . The transformation $G_{k}$ is defined by replacing the gauge-fixing delta function $\delta_{k, A x}(A)$ in (4.1.1) by the Landau-gauge fixing function $\mathscr{G}\left(\partial^{*} A\right)$ of (4.4.1). The operator $G_{k}$ was given in the momentum representation in [6I, Eqs. (1.83) and (1.84)]. Starting from this expression, one can derive the following formulas for $\sigma_{k}(p)$ by straightforward, algebraic manipulation:

We express $\sigma_{k}$ as a sum of two terms

$$
\sigma_{k}=\tau_{1}+\tau_{2} .
$$

Here $\tau_{1}$ vanishes on curls. Thus if $f=\partial B$, then $\tau_{1} f=0$. Explicitly

$$
\tau_{1, \mu v \lambda \alpha}\left(p^{\prime}\right)=\frac{1}{2} \sum_{l}\left(\frac{|u|^{2}}{\bar{v}_{\mu} \bar{v}_{v} v_{\lambda} v_{\kappa}}\left[\delta_{\mu \lambda}-\frac{\partial_{\mu} \bar{\partial}_{\lambda}}{\Delta}\right]\left[\delta_{v \kappa}-\frac{\partial_{v} \bar{\partial}_{\kappa}}{\Delta}\right]\right)\left(p^{\prime}+l\right),
$$


and

$$
\tau_{2, \mu \nu \lambda \kappa}\left(p^{\prime}\right)=\left(a_{\mu} \bar{a}_{\lambda}\left(\varphi_{v} \varphi_{\kappa}\right)^{-1 / 2}\left[\delta_{v \kappa}-\frac{\partial_{v}^{(1)} \overline{\partial_{\kappa}^{(1)}}}{\sqrt{\varphi_{v} \varphi_{\kappa}}}\left(\sum_{\varrho}\left|\partial_{Q}^{(1)}\right|^{2} / \varphi_{Q}\right)^{-1}\right]\right)\left(p^{\prime}\right) .
$$

In the formula for $\tau_{1}\left(p^{\prime}\right)$, each term on the right side is evaluated at momentum $p^{\prime}+l$. In the formula for $\tau_{2}$, the averaging only occurs in

$$
a_{\mu}\left(p^{\prime}\right)=\partial_{\mu}^{(1)}(p) \sum_{l}\left(\left|\frac{u}{v_{\mu}}\right|^{2} \frac{1}{\Delta}\right)\left(p^{\prime}+l\right) .
$$

In both $\tau_{1}$ and $\tau_{2}$ the expressions inside brackets [ ] are projection operators. Thus $\sigma_{k}$ has the general form of a sum of projection operators, or tensor products of projection operators, sandwiched between averaging operators.

The fact that $\tau_{1}$ vanishes on curls can be established as follows: The general form of $\tau_{1}$ in configuration space is evident from (7.1.11), (7.1.14) namely

$$
\tau_{1}=Q_{k}^{e}\left(I-P_{\partial}\right) Q_{k}^{e *},
$$

where $P_{\partial}$ denotes the orthogonal projection onto curls. (If $P_{1}$ projects one-forms onto gradients, then $P_{1} \otimes P_{1}$ projects two forms onto curls.) Furthermore $Q_{k}^{e *} \partial=\partial Q_{k}^{s *}$, so

$$
\tau_{1} \partial=Q_{k}^{e}\left(I-P_{\partial}\right) \partial Q_{k}^{s *}=0
$$

Let $\mathscr{K}(p)$ denote the space of momentum $p$ lattice one-forms $B$. Write the twoforms $\mathscr{H}(p)$ as a sum of curls and an orthogonal complement,

$$
\mathscr{H}(p)=\partial \mathscr{K}(p)+(\partial \mathscr{K}(p))^{\perp}
$$

Here we now introduce the scalar product,

$$
(f, g)=\sum_{\mu, v} \overline{f_{\mu v}(p)}\left|v_{\mu}(p) v_{v}(p)\right|^{-2} g_{\mu v}(p)=\left\langle f,|V|^{-2} \otimes|V|^{-2} g\right\rangle
$$

and the decomposition (7.1.19) is with respect to this inner product. This decomposition is natural in the study of $\sigma_{k}(p)$, since $\tau_{1}(p) \partial \mathscr{K}(p)=0$.

Remark that there are strictly positive constants $c_{1}, c_{2}$ such that

$$
c_{1}\langle f, f\rangle \leqq(f, f) \leqq c_{2}\langle f, f\rangle,
$$

so the corresponding norms are equivalent. In fact

$$
\left|v_{\mu}(p)\right|=\left[\frac{\sin \left(p_{\mu} / 2\right)}{\left(p_{\mu} / 2\right)}\right]\left[\frac{\left(p_{\mu} \eta / 2\right)}{\sin \left(p_{\mu} \eta / 2\right)}\right]
$$

and since for $|x| \leqq \pi / 2$,

$$
\frac{2}{\pi} \leqq \frac{\sin x}{x} \leqq 1
$$

it follows that for $\left|p_{i}\right| \leqq \pi$,

$$
\frac{2}{\pi} \leqq\left|v_{\mu}(p)\right| \leqq \frac{\pi}{2}
$$


Proposition 7.1.2. Suppose there exists $\varepsilon>0$, and $\tau_{0}(p) \leqq \tau_{1}(p)$ such that for $\left|p_{j}\right| \leqq \pi$,

$$
\varepsilon \leqq \tau_{0}(p)\left\lceil(\partial \mathscr{K}(p))^{\perp} \text { and } \varepsilon \leqq \tau_{2}(p)\lceil\partial \mathscr{K}(p) .\right.
$$

Then Theorem 7.1.1 holds.

Proof. It is sufficient to show that there is a constant $c>0$ such that

$$
c \leqq \sigma_{k}(p)
$$

for all $\left|p_{j}\right| \leqq \pi$. The inequality

$$
\left|a_{\mu}(p) / \varphi_{\nu}(p)^{1 / 2}\right| \leqq c_{2}
$$

is an extension of (7.1.20). As a consequence,

$$
\left\|\tau_{2}\right\| \leqq M
$$

where $M$ is a constant independent of $p$ and $k$.

Let us now decompose $\sigma_{k}(p)$ according to (7.1.19). For $f=\partial B+f^{\perp} \in \mathscr{H}(p)$, write

$$
\begin{aligned}
\left\langle f, \sigma_{k} f\right\rangle & =\left\langle f, \tau_{1} f\right\rangle+\left\langle f, \tau_{2} f\right\rangle \\
& =\left\langle f^{\perp}, \tau_{1} f^{\perp}\right\rangle+\left\langle f, \tau_{2} f\right\rangle \geqq\left\langle f^{\perp}, \tau_{0} f^{\perp}\right\rangle+\left\langle f, \tau_{2} f\right\rangle \\
& \geqq \varepsilon\left\|f^{\perp}\right\|^{2}+\left\langle f, \tau_{2} f\right\rangle .
\end{aligned}
$$

Here we use $\tau_{1} \partial=0$ and the hypothesis on $\tau_{1}$. The idea is to bound $\left\langle f, \tau_{2} f\right\rangle$ using the positivity of $\tau_{2}$. In fact, $0 \leqq \tau_{2}$ is evident from (7.1.15). Then for any $\delta \in[0,1]$,

$$
\left\langle f, \sigma_{k} f\right\rangle \geqq \varepsilon\left\|f^{\perp}\right\|^{2}+\delta\left\langle f, \tau_{2} f\right\rangle .
$$

Note that by (7.1.24) and the positivity of $\tau_{2}$,

$$
\begin{aligned}
\left\langle f, \tau_{2} f\right\rangle & =\left\langle\partial B, \tau_{2} \partial B\right\rangle+\left\langle\partial B, \tau_{2} f^{\perp}\right\rangle+\left\langle f^{\perp}, \tau_{2} \partial B\right\rangle+\left\langle f^{\perp}, \tau_{2} f^{\perp}\right\rangle \\
& \geqq\left\langle\partial B, \tau_{2} \partial B\right\rangle-2 M\|\partial B\|\left\|f^{\perp}\right\| .
\end{aligned}
$$

For any $\lambda>0$, we then have

$$
\left\langle f, \tau_{2} f\right\rangle \geqq\left\langle\partial B, \tau_{2} \partial B\right\rangle-(M \lambda)^{2}\|\partial B\|^{2}-\lambda^{-2}\left\|f^{\perp}\right\|^{2} .
$$

Use the lower bound (7.1.20) on $\tau_{2} 1 \partial \mathscr{K}$, and choose $\lambda$ so $(M \lambda)^{2}=\varepsilon / 2$. Thus

$$
\left\langle f, \tau_{2} f\right\rangle \geqq \frac{1}{2} \varepsilon\|\partial B\|^{2}-2 M^{2} \varepsilon^{-1}\left\|f^{\perp}\right\|^{2} .
$$

Inserting this in (7.1.26) yields

$$
\left\langle f, \sigma_{k} f\right\rangle \geqq\left(\varepsilon-2 M^{2} \delta \varepsilon^{-1}\right)\left\|f^{\perp}\right\|^{2}+\frac{1}{2} \delta \varepsilon\|\partial B\|^{2} \geqq \frac{1}{2} \varepsilon\left\|f^{\perp}\right\|^{2}+\frac{1}{2} \delta \varepsilon\|\partial B\|^{2},
$$

where the last inequality follows for any $\delta<(\varepsilon / 2 M)^{2} \leqq 1$. Let $\delta>0$ and $c \equiv \frac{1}{2} \delta \varepsilon>0$. Then

$$
\left\langle f, \sigma_{k} f\right\rangle \geqq c\left(\left\|f^{\perp}\right\|^{2}+\|\partial B\|^{2}\right)=c\|f\|^{2},
$$

as desired and completes the proof of the proposition.

Let us now prove the lower bound (7.1.21) on $\tau_{0}(p)$. Introduce the operator $\tau_{0}(p)$ obtained by restricting the sum in (7.1.14) to $l=0$. Clearly each term in the 
sum over $l$ is a nonnegative operator. Hence

$$
\tau_{0}(p) \leqq \tau_{1}(p)
$$

Note that because $f^{\perp}$ is defined with respect to the inner product $(7.1 .19 b), \tau_{0}$ simplifies on $f^{\perp}$. In particular the $\partial$ terms vanish and

$$
\left\langle f^{\perp}, \tau_{0} f^{\perp}\right\rangle=\frac{1}{2}|u(p)|^{2} \sum_{\mu, v}\left|f_{\mu \nu}^{\perp}(p) /\left(v_{\mu}(p) v_{v}(p)\right)\right|^{2} .
$$

From (7.1.20) we then infer

$$
\varepsilon \leqq \tau_{0}\left\lceil(\partial \mathscr{K})^{\perp},\right.
$$

for some $\varepsilon>0$, which with (7.1.28) yields the desired lower bound

$$
\varepsilon \leqq \tau_{1} \Gamma(\partial \mathscr{H})^{\perp} .
$$

Next we consider the bound on $\tau_{2} \uparrow \partial \mathscr{K}$. But since $\tau_{1} \partial=0$,

$$
\left\langle\partial B, \tau_{2} \partial B\right\rangle=\left\langle\partial B, \sigma_{k} \partial B\right\rangle=\left\langle B, \Delta_{k} B\right\rangle,
$$

using the definition (4.3.1) of $\Delta_{k}$. An explicit formula for $\Delta_{k}$ shows that

$$
\left\langle\partial B, \sigma_{k} \partial B\right\rangle=\frac{1}{2}\left(\sum_{Q}\left|\partial_{Q}^{(1)}\right|^{2} / \varphi_{e}\right)^{-1} \sum_{\mu, v}\left|(\partial B)_{\mu \nu}\right|^{2} /\left(\varphi_{\mu} \varphi_{v}\right) .
$$

which is bounded below by $\varepsilon\|\partial B\|^{2}$ (see also $[6 \mathrm{I}, 8]$ ). This completes the proof of Theorem 7.1.1.

\subsection{Regularity and Decay of Propagators and Minimizers}

We are interested in Landau gauge propagators and minimizers, because they have good regularity and decay. The key objects are $H_{k}$ and $C^{(k)}$, while estimates on $\mathscr{D}_{k}$ follow by (4.4.4). The minimizer $H_{k}$ can be expressed as an integral kernel. For $x \in T_{\eta}$

$$
\left(H_{k} B\right)_{\mu}(x)=\sum_{y \in T_{1}^{(k)}, v} H_{k, \mu \nu}(x ; y) B_{v}(y)
$$

The kernel $H_{k, \mu v}(x, y)$ and its gradient decay exponentially. In particular there exists $\delta>0$ and for $0 \leqq \alpha<1$ a constant $M=M(\alpha)<\infty$ such that for $\left|x-x^{\prime}\right| \leqq 1$,

$$
\begin{aligned}
\left|H_{k, \mu v}(x, y)\right| & +\left|\nabla H_{k, \mu v}(x, y)\right| \\
& +\left|x-x^{\prime}\right|^{-\alpha}\left|\nabla H_{k, \mu \nu}(x, y)-\nabla H_{k, \mu \nu}\left(x^{\prime}, y\right)\right| \leqq M e^{-\delta|x-y|} .
\end{aligned}
$$

This inequality is a consequence of Proposition 1.2 and the representation (1.103) of $[61]$.

The unit lattice propagator $C^{(k)}$ also has exponential decay,

$$
\left|C_{\mu \nu}^{(k)}(x, y)\right| \leqq M e^{-\delta|x-y|},
$$

for $x, y \in T_{1}^{(k)}$. This inequality follows from the bound (2.157) in [6II] and from the general theorem on unit lattice operators in [7]. In fact this inequality also holds for propagators with Dirichlet boundary conditions outside a domain $A$, uniformly in $\Lambda$. 
The gauge transformation $\lambda$ in (5.1.1) is bounded and depends on $B$ through an exponentially decaying kernel $D_{k}$ :

$$
\lambda(x)=\left(D_{k} B\right)(x),\left|D_{k}(x, b)\right| \leqq M e^{-\delta \operatorname{dist}(x, b)} .
$$

This estimate follows from (5.1.4) and (7.2.2).

The operators $\mathscr{D}_{k}$ have the same properties as the operators $G_{k}$ in [6I], Proposition 1.2, with exponential decay but singularities on the diagonal. These properties follow from (4.4.4) and the above estimates on $H_{k}, C^{(k)}$.

\subsection{Positivity of $\Delta_{k}\left(u_{k}\right)$}

The scalar field quadratic form $A_{k}\left(u_{k}\right)$ depends on the background field $u_{k}$. Hence we can only establish stability properties for $\Delta_{k}\left(u_{k}\right)$ with some restriction on $u_{k}$. In particular, let us assume that for the unit lattice field $v$,

$$
|v(\partial p)-1| \leqq e_{k} \not\left(e_{k}\right),
$$

where $\not 2\left(e_{k}\right)=\left(1+\ln e_{k}^{-1}\right)^{\prime}$. Then the stability estimate can be stated in two forms. For constants $\gamma>0, \alpha>0, M<\infty$,

$$
\left\langle\phi, \Delta_{k}\left(u_{k}\right) \phi\right\rangle \geqq \gamma \sum_{b \in T_{1}^{(k)}}\left|u_{k}(b) \phi\left(b_{+}\right)-\phi\left(b_{-}\right)\right|^{2}-M e_{k}^{2-\alpha} \sum_{x \in T_{1}^{(k)}}|\phi(x)|^{2} .
$$

The second form of the inequality substitutes $v_{b}$ for $u_{k}(b)$ in the covariant derivative of $\phi$. These inequalities can be proved by an extension of the proofs of [7]. The propagators arising from $\Delta_{k}\left(u_{k}\right)$, under the restriction (7.3.1) on the gauge field, also satisfy the regularity and decay estimates of [7]. In order to remain within the framework of this reference, we remark that by change of gauge $u_{k}$ can be transformed in a local region $\Lambda$ into a configuration of the form $\exp \left[i e_{k} \eta A\right]$, where $A$ is smooth and small. In axial gauge for the configuration $v$ in a domain $A^{\prime} \supset \Lambda$ we can substitute $\mathscr{D}_{k} \partial^{*}=G_{k, A x} \partial^{*}+\partial D$ in the formula for $u_{k}$, we use (5.3.1) to replace this by a minimizer in axial gauge. Then we use (5.1.1) to return the minimizers to Landau gauge. This is a local procedure since $f^{(k)}$ can locally be represented as a curl.

\section{Appendix. Quadratic Forms}

In the analysis of this paper we often wish to minimize a quadratic form on a Hilbert space $\mathscr{H}$, possibly subject to a constraint. We collect here a couple of elementary properties which we use, and which could serve as the start of a more general study of related problems.

Since we work with lattice fields, our space $\mathscr{H}$ is finite dimensional and all forms are bounded. Of course we may obtain limiting unbounded forms as $\operatorname{dim} \mathscr{H}$ $\rightarrow \infty$ (e.g., as in infinite-volume or zero-lattice-spacing limit). Such forms are analyzed as limits of finite-dimensional approximations.

As a first example, let $0 \leqq \Delta=\alpha^{*} \alpha$ be a self-adjoint transformation on $\mathscr{H}$ and let $B \in \mathscr{H}$. Define the quadratic form

$$
h(A)=\frac{1}{2}\|\alpha A+B\|^{2} .
$$

Let $P$ denote the projection onto Range $(\alpha)$. 
Proposition A1. Range $\alpha^{*}=$ Domain $A^{-1}$, so $P=\alpha A^{-1} \alpha^{*}$. Furthermore

$$
h(A) \geqq h\left(A_{c l}\right)=\frac{1}{2}\langle B,(I-P) B\rangle,
$$

where

$$
A_{c l}=-\Delta^{-1} \alpha^{*} B
$$

is the unique minimum of (A1).

Proof. Note that Range $\alpha^{*}=(\operatorname{Kernel} \alpha)^{\perp}$ and Kernel $\alpha=\operatorname{Kernel} \Delta$. By the spectral theorem $\mathscr{H}=\operatorname{Kernel}(\Delta) \oplus \operatorname{Range}(\Delta)$, so $\operatorname{Range} \alpha^{*}=\operatorname{Range} \Delta=\operatorname{Domain} \Delta^{-1}$. Therefore $P=\alpha A^{-1} \alpha^{*}$ exists, $P^{2}=P=P^{*}$, and $P \alpha=\alpha$. Thus $P$ is the projection onto Range $\alpha$. Writing

$$
h(A)=\frac{1}{2}\left\|A^{1 / 2} A+A^{-1 / 2} \alpha^{*} B\right\|^{2}+\frac{1}{2}\langle B,(I-P) B\rangle
$$

yields (A2). Since $h(A)$ is convex, the minimum is unique, to complete the proof.

If we translate the quadratic form $h$, using the change of variables

$$
A=A^{\prime}+A_{c l},
$$

then as a consequence of $(I-P) \alpha=0$, and $h\left(A_{c l}\right)=\frac{1}{2}\langle B,(I-P) B\rangle, h(A)$ decomposes into a sum of independent quadratic forms. We state

Corollary A2. With $h(A)$ given by (A1) and $A=A^{\prime}+A_{c l}$ defined by (A3),

$$
h(A)=\frac{1}{2}\left\langle A^{\prime}, \Delta A^{\prime}\right\rangle+h\left(A_{c l}\right) .
$$

We now specialize to the case where $\mathscr{H}=L_{2}\left(\mathbb{R}^{N}, d x\right)$, with $d x$ Lebesgue measure. We consider the Green's function $C$ of $\Delta$. Assuming $0<A$, then we have two convenient representations for $C$. First

$$
C=\Delta^{-1} \text {, }
$$

by which we express $C$ as the operator inverse of $\triangle$. Secondly, we can express $C$ as a moment of a Gaussian measure, namely

$$
\exp \left(\frac{1}{2}\langle B, C B\rangle\right)=Z^{-1} \int_{\mathscr{H}} \exp \left(-\frac{1}{2}\langle x, \Delta x\rangle+\langle x, B\rangle\right) d x
$$

We are often interested, however, in the case of operators $\Delta$ with zero modes. Thus $\Delta$ has an inverse only when restricted to a subspace $\mathscr{H}_{0} \subset \mathscr{H}$ which does not contain zero modes. Likewise an integral such as (A6) converges only when restricted to such a subspace. In gauge theories it is often natural to choose various subspaces $\mathscr{H}_{0}$, and furthermore $\Delta$ is generally not diagonal under the decomposition $\mathscr{H}=\mathscr{H}_{0} \oplus \mathscr{H}_{0}^{\perp}$. Thus we are led to study quadratic forms $\Delta$ constrained to act on $\mathscr{H}_{0}$.

Define the transformation $C$ on $\mathscr{H}$ by

$$
\exp \left(\frac{1}{2}\langle B, C B\rangle\right)=Z^{-1} \int_{\mathscr{H}_{0}} \exp \left(-\frac{1}{2}\langle x, \Delta x\rangle+\langle x, B\rangle\right) d x .
$$

Clearly $C$ is self-adjoint in the inner product $\langle$,$\rangle . Furthermore, define$

$$
\Delta_{0}=\Delta \uparrow \mathscr{H}_{0} \text {. }
$$


In general $\mathscr{H}_{0} \neq \mathscr{H}$ and $C \uparrow \mathscr{H}_{0} \neq \Delta_{0}^{-1}$. Thus we ask

(1) How are $\Delta_{0}$ and $C$ related?

(2) What is the minimizer of the quadratic form $\frac{1}{2}\left\langle x, \Delta_{0} x\right\rangle-\langle x, B\rangle$ for $x \in \mathscr{H}_{0}$ ?

Let us make the following assumptions: Let $V$ be given and

$$
\begin{gathered}
I \leqq \Delta_{0} \quad \text { on } \quad \mathscr{H}_{0}, \\
I \leqq \Delta+V \quad \text { on } \mathscr{H},
\end{gathered}
$$

where $\mathscr{H}_{0}=$ Null $V$. With these definitions, define

$$
G=(\Delta+V)^{-1}
$$

and let $($,$) be the inner product$

$$
(x, y) \equiv\langle x,(\Delta+V) y\rangle .
$$

Then let $P$ denote the projection of $\mathscr{H}$ onto $\mathscr{H}_{0}$, where $P$ is orthogonal in the inner product (A12).

Proposition A3. Under the assumptions above,

$$
C=P G,
$$

so Range $C \subset \mathscr{H}_{0}$. Furthermore

and

$$
\begin{gathered}
C \Delta_{0} C=C, \\
H B=C B=Z^{-1} \int_{\mathscr{H}_{0}} \exp \left(-\frac{1}{2}\langle x, \Delta x\rangle+\langle x, B\rangle\right) x d x .
\end{gathered}
$$

is the minimum configuration of

$$
\frac{1}{2}\langle x, \Delta x\rangle-\langle x, B\rangle, \quad x \in \mathscr{H}_{0} .
$$

Thus with $x=y+C B$,

$$
\frac{1}{2}\langle x, \Delta x\rangle-\langle x, B\rangle=\frac{1}{2}\langle y, \Delta y\rangle-\frac{1}{2}\langle B, C B\rangle, \quad x \in \mathscr{H}_{0} .
$$

Proof. For $x \in \mathscr{H}_{0}$, note that

$$
\frac{1}{2}\left\langle x, \Delta_{0} x\right\rangle-\langle x, B\rangle=\frac{1}{2}\langle x,(A+V) x\rangle+\langle x,(A+V) G B\rangle .
$$

Thus (A7) can be written

$$
\begin{aligned}
\exp \left(\frac{1}{2}\langle B, C B\rangle\right)= & Z^{-1} \int_{\mathscr{H}_{0}} \exp \left(-\frac{1}{2}\langle x-P G B,(\Delta+V)(x-P G B)\rangle\right. \\
& \left.+\frac{1}{2}\langle P G B,(\Delta+V) P G B\rangle\right) d x,
\end{aligned}
$$

where we have used

$$
\langle P G B,(\Delta+V) x\rangle=(P G B, x)=(G B, P x)=(G B, x)=\langle G B,(\Delta+V) x\rangle .(A
$$

Thus (A17) equals

$$
\exp \left(\frac{1}{2}\langle P G B,(\Delta+V) P G B\rangle\right)=\exp \left(\frac{1}{2}\langle G B,(\Delta+V) P G B\rangle\right)=\exp \left(\frac{1}{2}\langle B, P G B\rangle\right) .
$$

Thus $\langle B, C B\rangle=\langle B, P G B\rangle$, and (A 13) holds as claimed. To verify (A14), note that

$$
C \Delta_{0} C=P G \Delta_{0} P G=P G(\Delta+V) P G=P^{2} G=P G=C .
$$


Next note that (A17) displays $C B$ as the minimum of $\frac{1}{2}\langle x, \Delta x\rangle-\langle x, B\rangle$, since linear terms in the fluctuation $x-P G B$ do not occur. Also from (A18) we have for $x \in \mathscr{H}_{0}$,

$$
\langle C B, \Delta x\rangle=\langle B, x\rangle,
$$

so the integral in (A15) equals $C B$. Finally, for $x \in \mathscr{H}_{0}$, so by (A19), $\langle C B, \Delta y\rangle$ $\equiv\langle B, y\rangle$. It follows that

$$
\begin{aligned}
\frac{1}{2}\langle x, A x\rangle-\langle x, B\rangle & =\frac{1}{2}\langle y, \Delta y\rangle+\langle\Delta y, C B\rangle+\frac{1}{2}\langle C B, \Delta C B\rangle-\langle y, B\rangle-\langle C B, B\rangle \\
& =\frac{1}{2}\langle y, \Delta y\rangle-\frac{1}{2}\langle B, C B\rangle,
\end{aligned}
$$

which is (A 16).

\section{References}

1. Bałaban, T., Imbrie, J., Jaffe, A.: Exact renormalization group for gauge theories, 1983 Cargese Lectures, Progress in Gauge Field Theories, pp. 79-104. G. 't Hooft et al. (ed.). New York: Plenum Press 1984

2. Brydges, D., Fröhlich, J., Seiler, E.; On the construction of quantized gauge fields. I. General results. Ann. Phys. 121, 227-284 (1979). II. Convergence of the lattice approximation. Commun. Math. Phys. 71, 159-205 (1980). III. The two-dimensional abelian Higgs model without cutoffs. Commun. Math. Phys. 79, 353-400 (1981)

3. Bałaban, T.: (Higgs) $)_{2,3}$ quantum fields in a finite volume. I. A lower bound. Commun. Math. Phys. 85, 603-626 (1983). II. An upper bound. Commun. Math. Phys. 86, 555-594 (1982)

4. King, C.: The U(1) Higgs model. I. The continuum Limit, HUTMP 84/B 167. II. The infinite volume limit. HUTMP 84/B168

5. Bałaban, T., Brydges, D., Imbrie, J., Jaffe, A.: The mass gap for Higgs models on a unit lattice. Ann. Phys. 158, 281-319 (1984)

6. Bałaban, T.: Propagators and renormalization transformations for lattice gauge theories. I. Commun. Math. Phys. 95, 17-40 (1984). II. Commun. Math. Phys. 96, 223-250 (1984)

7. Bałaban, T.: Regularity and decay of lattice Green's functions. Commun. Math. Phys. 89, 571-597 (1983)

8. Federbush, P.: A phase cell approach to Yang-Mills theory. I. Small field modes. University of Michigan preprint

Communicated by A. Jaffe

Received October 26, 1984 